Indian Journal of Science and Technology

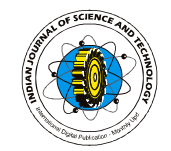

\title{
Second order constraints in the theory of invariant relative orbits including relativistic and direct solar radiation pressure effects
}

\author{
F A Abd El-Salam ${ }^{1}$ and M I El-Saftawy ${ }^{2}$ \\ ${ }^{1}$ Department of Mathematics, Taibah University, Faculty of Science, Al-Madeenah Al-Monawwarah, K.S.A \\ ${ }^{2}$ Department of Astronomy and Space, King Abdul-Aziz University, Faculty of Science, Jeddah, K.S.A \\ 1f.a.abdelsalam@gmail.com, ${ }^{2}$ malsaftawy@kau.edu.sa
}

\begin{abstract}
Working with the mean orbit elements, the secular drift of the longitude of the ascending node and the sum of the argument of perigee and mean anomaly are set equal between two neighboring orbits. By having both orbits drift at equal angular rates on the average, they will not separate over time due to the influence of the perturbative effects of the asphericity of the Earth. The relativistic corrections and the direct solar radiation pressure to the equations of motion, as is considered in this work. The problem is stated. The expressions for the time rate of change of the longitude of the ascending node and the sum of the argument of perigee and mean anomaly in terms of the augmented Delaunay canonical elements are obtained. The expressions for the second order conditions that guarantee that the drift rates of two neighboring orbits are equal on the average are derived.
\end{abstract}

Keywords: Invariant relative orbits, Spacecraft flying formations, Relativistic corrections, Direct solar radiation pressure. Introduction

The formation flying concept is the use of several small satellites which work together in a group to accomplish the objective of one larger, usually more expensive, satellite. This actually increases the likelihood of mission success in the event of a malfunction. Although the benefits are numerous, formation flying missions have many difficulties that single platform missions do not. A static formation, in which there is no relative motion between spacecrafts and is only possible for satellites in circular, coplanar orbits. For non-coplanar, non circular orbits there is relative motion in both the longtrack and cross-track directions (Hughes, 1999).

The problem of designing a satellite formations applies all of the criteria for designing a single satellite orbit. In fact orbit design has no absolute rules. Effective orbit design requires clearly identifying the reasons for orbit selection, reviewing these reasons regularly as mission requirements change or mission definition improves, and continuing to remain open to alternatives. Thus, it is needed to consider whether each satellite is launchable, survivable, and properly in view of ground stations or relay satellites. It is also required to consider the number of satellites, their relative positions, and how these positions change with time, both in the course of an orbit and over the lifetime of the formation. We will reference all the orbits of the spacecraft, namely the deputy orbits to one orbit in the Research article CIndian Society for Education and Environment (iSee)

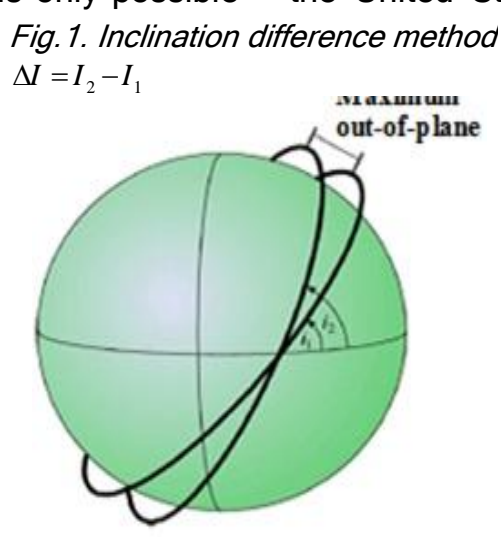

Fig. 2.Node difference method $\Delta \Omega=\Omega_{2}-\Omega_{1}$.

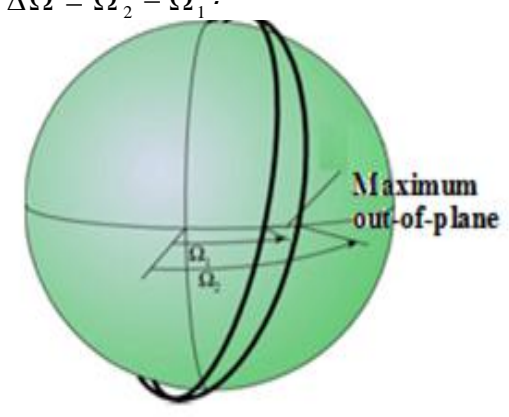

"Direct solar radiation pressure test" http://www.indjst.org formation namely the chief. The different methods to setup invariant relative orbits for spacecraft flying formations are: 1- Inclination difference method, 2- Node difference method, 3- Combination of the above two methods (see Fig. 1 and Fig. 2).

The literature is a wealth with works dealing with designing certain invariant relative orbits for spacecraft flying formations, and it seems worth to sketch some of the most relevant works. Easton and Brescia (1969) of the United States Naval Research Laboratory analyzed coverage by satellites in two mutually perpendicular orbit planes and concluded that we would need at least six satellites to provide complete Earth coverage. Alfriend, et al. (2001) presented two new concepts for minimizing fuel consumption for formation flying satellites. First they derived the changes in the orbital parameters for negating the out-ofplane drift and in track drift due to the $\mathrm{J}_{2} \quad$ perturbation. Second, they introduced a strategy for equalizing the fuel consumption over all the satellites in the constellation.

Schaub and Alfriend (2001) presented an analytical method to establish $J_{2}$ invariant relative orbits using the mean orbit elements. They obtained two first order conditions between the differences in momenta elements (semi-major axis $a$, eccentricity $e$, inclination $I$ ) that guarantee that the drift rates of two neighboring orbits are equal on the average. Secular drift of the longitude of

F.A.A.El-Salam \& M.I.EI-Saftawy Indian J.Sci.Technol. 
the ascending node and the sum of the argument of perigee and mean anomaly were set equal between two neighboring orbits. The resulting orbits require less control and maintenance fuel. Alfriend et al. (2002) addressed relatively motioning orbits for formation flying satellites considering $\mathrm{J}_{2}$ as the perturbing force only.

They categorized the various types of orbits according the number of constraints on the initial conditions of the orbital elements, the various kinds of the orbits in each category are identified and estimates given for fuel requirement to maintain a bounded orbit.

Since the differential gravity perturbations are functions of $(a, e, I)$, the small changes in these elements result in different drift rates for each satellite and the negation of these drifts result in different fuel requirements for each satellite. Since some satellites running out of fuel before others will degrade the system performance it would be advantageous to have the satellites having equal fuel consumption. Using the Hamiltonian framework, the authors aim to tackle the following points:

- Constructing an analytical method to design invariant relative orbits for the flying formation.

- The emphasis will be on secular oblateness perturbations due to the zonal harmonics $J_{2}, J_{3}, J_{4}$ and the relativistic corrections to the equations of motion.

- The invariance, regarding the perturbations considered, in the longitude of the ascending node and in mean argument of latitude are obtained up to the second order, assuming $J_{2}$ is of order 1 and $\frac{1}{c_{l}^{2}}$ is of order 2 .

\section{Hamiltonian of the problem}

Consider a satellite moves in Earth's gravitational field and perturbed by the direct solar radiation pressure. We can safely assume that the Sun moves in a circular orbit, under this assumption $f_{\mathrm{e}}+\omega_{\mathrm{e}}$ the Sun's true anomaly plus its argument of perigee reduces to the Sun's mean longitude $\Omega_{\mathrm{e}}$. Also we can assume that the direction and distance of the satellite from the Sun are similar to those of the Earth, i.e. $\left(r_{0} / r_{\mathrm{e}}\right)^{2} ; 1$ Utilizing these assumputions the Hamiltonian of the problem is constructed as;

$$
\mathrm{H}=\sum_{n=0}^{4} \frac{J_{2}^{n}}{n !} \mathrm{H}_{n}
$$

where $\mathrm{H}_{0}$ is the integrable part of the Hamiltonian, $\mathrm{H}_{n}, n \ldots 1$ are the perturbing Hamiltonians at diffetent orders, where we consider $J_{2}$ of order 1 and $\frac{1}{c_{l}}$ of order

2 , Thus we obtain

$\mathrm{H}_{0}=-\frac{\mu^{2}}{2 L^{2}}$,

$$
\begin{aligned}
& \mathrm{H}_{1}=\frac{\mu^{4} R^{2}}{4 L^{6}} \Psi^{3}\left[\left(3 S^{2}-2\right)-3 S^{2} \cos F_{2,2}\right]+A_{1} K, \\
& \mathrm{H}_{2}=\frac{1}{4} \frac{A_{1,2}}{L^{6}} \Psi^{3}\left[\left(3 S^{2}-2\right)-3 S^{2} \cos F_{2,2}\right] \\
& +\frac{1}{8} \frac{A_{2,3}}{L^{8}} \Psi^{4}\left[\left(15 S^{3}-12 S\right) \sin F_{1,1}-5 S^{3} \sin F_{3,3}\right] \\
& +\frac{1}{64} \frac{A_{2,4}}{L^{10}} \Psi^{5}\left\{\left(105 S^{4}-102 S^{2}+24\right)\right. \\
& \left.+\left(-140 S^{4}+120 S^{2}\right) \cos F_{2,2}+35 S^{4} \cos F_{4,4}\right\} \\
& +\frac{B_{2}}{L^{4}}\left[-3 \Psi^{2}+2 \Psi-\frac{1}{8}\right] \\
& \mathrm{H}_{3}=\frac{1}{4} \frac{B_{3,2}}{L^{8}}\left[2 \Psi^{4}-\frac{3}{2} \Psi^{3}\right] \\
& \left.-\left(18 S^{4}-12 S^{2}\right) \cos F_{2,2}+\frac{9}{2} S^{4} \cos F_{4,4}\right\} \\
& +\frac{1}{8} \frac{B_{4,3}}{L^{10}}\left[2 \Psi^{5}-\frac{3}{2} \Psi^{4}\right] \\
& \times\left[\left(15 S^{3}-12 S^{2}\right) \sin F_{1,1}-5 S^{3} \sin F_{3,3}\right] \\
& \left.+\left(-140 S^{4}+120 S^{2}\right) \cos F_{2,2}+35 S^{4} \cos F_{4,4}\right\} \\
& +\frac{1}{32} \frac{B_{5,23}}{L^{14}} \Psi^{7}\left[\left(60 S^{5}-84 S^{3}+24 S^{2}\right) \sin F_{1,1}\right. \\
& \left.-\left(\frac{75}{2} S^{5}-28 S^{3}\right) \sin F_{3,3}+\frac{15}{2} S^{3} \sin F_{5,5}\right] \\
& +\frac{1}{256} \frac{B_{5,24}}{L^{16}} \Psi^{8}\left[\left(525 S^{6}-750 S^{4}+312 S^{2}-48\right)\right. \\
& +\left(-\frac{1575}{2} S^{6}+1000 S^{4}-312 S^{2}\right) \cos F_{2,2} \\
& \left.+\left(315 S^{6}-250 S^{4}\right) \cos F_{4,4}-\frac{105}{2} S^{6} \cos F_{6,6}\right]
\end{aligned}
$$

ISSN: 0974- 6846

where $f$ is the satellite's true anomaly, $g, h$ are the argument of perigee, and the longitude of the node of the satellite's orbit respectively, $R_{\oplus}$ is the mean Earth's radius $a$ is the semi-major axis, $r$ is the satellite's raduis vector, $I$ is the orbital inclination, $\varepsilon$ is the obliquity of the ecliptic, $J_{2}, J_{3}, J_{4}$ are the zonal harmonic coefficients, $A_{2,2}^{S R P}=(A / m)\left(S_{0} / J_{2}^{2} c_{l}\right) \cos \gamma_{i}\left(1-\beta_{i}\right)$ where $S_{0}$ is the solar constant (corresponding to the mean Earth-Sun distance, $\gamma$ is the angle of incidence between the two unit vectors along the direction satellite-Sun, and along the normal to the satellite $i^{\underline{\text { th }}}$ surface, $\beta_{i}$ is the specular reflectivity for $i^{\text {th }}$ surface, $(A / m)$ is the area to mass ratio, $\Psi=(a / r)$, $F_{\alpha, \beta, i, j}=\alpha f+\beta g+i h+j k$, the coefficients $C_{i, j}$ are given by 
$C_{ \pm 1, \pm 1}=(1 \pm C)\left(1 \pm C_{\varepsilon}\right), \quad C_{0, \mp 1}= \pm 2 S S_{\varepsilon}$,

$C_{\varepsilon}=\cos \varepsilon, S_{\varepsilon}=\sin \varepsilon, C=\cos I, S=\sin I$

The $A_{i, j}$ dimensionless parameters are defined by

$$
\begin{array}{ll}
A_{1,2}=\mu^{4} R_{\oplus}^{2}, & A_{2,3}=\frac{2 \mu^{5} R_{\oplus}^{3} J_{3}}{J_{2}^{2}}, \\
A_{2,4}=\frac{2 \mu^{6} R_{\oplus}^{4} J_{4}}{J_{2}^{2}}, & B_{2}=\frac{2 \mu^{4} c_{l}^{-2}}{J_{2}^{2}}, \\
B_{3,2}=3 ! \frac{\mu^{6} R_{\oplus}^{2} J_{2}}{J_{2}^{3} c_{l}^{2}}, & B_{4,2}=4 ! \frac{\mu^{8} R_{\oplus}^{4} J_{2}^{2}}{J_{2}^{4} c_{l}^{2}}, \\
B_{4,4}=3 ! \frac{c_{l}^{-2} \mu^{8} R_{\oplus}^{4} J_{4}}{J_{2}^{4}}, & B_{4,3}=4 ! \frac{\mu^{7} R_{\oplus}^{3} J_{3}}{J_{2}^{4} c_{l}^{2}}, \\
B_{5,23}=5 ! \frac{c_{l}^{-2} \mu^{9} R_{\oplus}^{5} J_{2} J_{3}}{J_{2}^{5}}, & B_{5,24}=5 ! \frac{c_{l}^{-2} \mu^{10} R_{\oplus}^{6} J_{2} J_{4}}{J_{2}^{5}},
\end{array}
$$

\section{The Transformed Hamiltonian}

Using the usual Delaunay canonical-variables augmented by celestial mean longitude of the Sun $k=\Omega_{\mathrm{e}}$ and its canonical conjugate $K$ as;

$l, g, h, k, L, G, H, K)$ defined by,

$$
\begin{aligned}
& l=M, \quad g=\omega, \quad h=\Omega, \quad k=\Omega_{\mathrm{e}} \\
& L=\sqrt{\mu a}, \quad G=L \sqrt{1-e^{2}}, \quad H=G \cos I, \quad K
\end{aligned}
$$

We used a perturbation technique based on Lie series and Lie transform, Kamel (1969), to eliminate the short as well as the long periodic terms, respectively from the Hamiltonian (3). We obtained the transformed Hamiltonian, for different orders 0, 1, 2, as (Abd ElSalam et al., 2005; El-Saftawy et al., 1998)

$$
\mathrm{H}^{* *}=\mathrm{H}_{0}^{* *}+\mathrm{H}_{1}^{* *}+\mathrm{H}_{2}^{* *}+\mathrm{H}_{3}^{* *}
$$

with

$$
\begin{aligned}
\mathrm{H}_{0}^{* *} & =-\frac{\mu^{2}}{2 L^{2}} \\
\mathrm{H}_{1}^{* *} & =\frac{1}{4} A_{1,2} \eta_{3,3}\left(1-3 C^{2}\right)+A_{1} K \\
\mathrm{H}_{2}^{* *} & =\frac{3}{64} \frac{A_{1,2}^{2}}{\mu^{2}}\left\{\left[5-10 C^{2}-35 C^{4}\right] \eta_{3,7}-\left[5-18 C^{2}+5 C^{4}\right] \eta_{5,5}\right\} \\
& +\frac{3}{128} A_{2,4}\left\{[ 1 5 - 1 5 0 C ^ { 2 } + 1 7 5 C ^ { 4 } ] \eta _ { 3 , 7 } \left[9-90 C^{2}\right.\right. \\
& \left.\left.+105 C^{4}\right] \eta_{5,5}\right\}-3 B_{2}\left\{\eta_{3,1}-\frac{5}{8} \eta_{4,0}\right\}-A_{2,2}^{S R P}\left(1+\frac{e^{2}}{2}\right)_{-2,0} \\
\mathrm{H}_{3}^{* *} & =B_{3,2}\left(3 S^{2}-2\right)\left\{\frac{3}{4} \eta_{3,5}-\frac{5}{8} \eta_{5,3}\right\}+\frac{B_{2} A_{1,2}}{\mu^{2}}\left(3 S^{2}-2\right) \\
& \times\left\{\frac{45}{4} \eta_{3,5}+\frac{27}{4} \eta_{4,4}-9 \eta_{5,3}\right\}-3\left(\frac{A_{11} A_{23}}{\mu^{2}}\right)_{g s}\left(3 \mathrm{~S}^{2}-2\right)
\end{aligned}
$$

Vol. 5 No. 9 (Sep. 2012)

ISSN: 0974- 6846

$$
\begin{aligned}
& \times\left\{\frac{1}{16} \eta_{7,-5}\left(100 \mathrm{e}^{2}-83 \mathrm{e}^{4}-2 \mathrm{e}^{6}\right)+\frac{1}{8}\left[2 \mathrm{e}^{4} \eta_{6,-4}+6 \mathrm{e}^{2} \eta_{4,-2}\right.\right. \\
& +\left(-6 \eta_{4,-2}-16 \eta_{3,-1}-9\right)-\frac{1}{3} \mathrm{e}^{-2}\left(11 \eta_{6,-4}-9 \eta_{4,-2}\right. \\
& \left.\left.-12 \eta_{3,-1}+36-36 \eta_{1,1}+15 \eta_{0,2}\right)\right]-\frac{1}{8} \eta_{5,-3}(-24 \\
& \left.+70 \mathrm{e}^{2}-44 \mathrm{e}^{4}\right)+\frac{3}{8} \eta_{4,-2}-\frac{3}{2} \eta_{3,-1}-\mathrm{e}^{2}\left(\frac{7}{4} \eta_{3,-1}\right. \\
& \left.\left.+6 \eta_{0,2}+3 \eta_{-2,4}\right)\right\}\left(\eta_{0,-2}-\eta_{-2,0}\right)^{-1}\left[\left(10 \eta_{-4,6}+12 \eta_{-3,5}\right.\right. \\
& +51 \eta_{-2,4}+228 \eta_{-1,3}-123 \eta_{0,2}-684 \eta_{1,1} \\
& +723 \eta_{2,0}+348 \eta_{3,-1}-705 \eta_{4,-2}+6 \eta_{6,-4} \\
& \left.\left.+144 \eta_{6,-4}\right)\right]+\frac{27 A_{11}\left(A_{2,2}^{S R P}\right)^{2}}{64 A_{12}^{2}}\left\{\left(1+C_{\varepsilon}^{2}+2 S_{\varepsilon}^{2}\right)\right. \\
& \left.\times\left(3 \eta_{0,-2}-4 \eta_{-2,0}\right)\right\} \\
& \times\left(\eta_{1,3}-2 \eta_{-1,5}\right)+2 H^{2}\left[3\left(1+C_{\varepsilon}^{2}+6 S_{\varepsilon}^{2}\right) \eta_{-1,7}\right. \\
+ & \left.\left(1+C_{\varepsilon}^{2}-12 S_{\varepsilon}^{2}\right) \eta_{0,-2}\right]-5 H^{4}\left(1+C_{\varepsilon}^{2}-2 S_{\varepsilon}^{2}\right) \\
& +L^{-i} G^{-j}
\end{aligned}
$$

\section{Statement of the problem}

To design an invariant relative orbits for spacecraft flying formations we follow:-

1. Compute the secular drift of the longitude of the ascending node $\dot{h}=\dot{\Omega}$, and the sum of the argument of perigee and mean anomaly $l+g$.

2. These secular drift rates are set equal between two neighboring orbits.

3. Having both orbits drift at equal angular rates on the average, they will not separate over time due to the influence of the perturbative effects of the asphericity of the Earth, the relativistic and the direct solar radiation pressure corrections to the equations of motion. Using the canonical equations of motion

$\dot{u}=\frac{\partial \mathrm{H}^{* *}}{\partial U} \quad \dot{U}=-\frac{\partial \mathrm{H}^{* *}}{\partial u}$

where $(u=l, g, h ; k \quad U=L, G, H, K)$. Substituting the transformed Hamiltonian (4) into equation (5) yields

$\dot{u}=\frac{\partial \mathrm{H}_{0}^{* *}}{\partial U}+J_{2} \frac{\partial \mathrm{H}_{1}^{* *}}{\partial U}+\frac{J_{2}^{2}}{2} \frac{\partial \mathrm{H}_{2}^{* *}}{\partial U}+\frac{J_{2}^{3}}{3 !} \frac{\partial \mathrm{H}_{3}^{* *}}{\partial U}$

Since the argument of mean latitude $(\theta)$ is the sum of the mean anomaly and the argument of perigee $(\theta=l+g)$. Evaluating the derivatives yields the sum of the argument of perigee and mean anomaly, $\theta$ and the secular drift rates of the longitude of the ascending node, 
Indian Journal of Science and Technology

$$
\dot{\boldsymbol{\theta}}=\sum_{n=0}^{3} \frac{J_{2}^{n}}{n !} \mathrm{D}_{n}^{\theta}, \quad \dot{h}=\sum_{n=1}^{3} \frac{J_{2}^{n}}{n !} \mathrm{D}_{n}^{h}
$$

With

$$
\begin{aligned}
\mathrm{D}_{0}^{\theta} & =\mu^{2} \bar{K}_{0}, \\
\mathrm{D}_{1}^{\theta} & =\frac{A_{1,2}}{4} \sum_{n=1}^{2} \bar{K}_{n}, \\
\mathrm{D}_{1}^{h} & =\frac{A_{1,2}}{4} Z_{1}, \\
\mathrm{D}_{2}^{h} & =\frac{3}{64} \frac{A_{1,2}^{2}}{\mu^{2}} \sum_{n=2}^{4} Z_{n}+\frac{3}{128} A_{2,4} \sum_{n=5}^{6} Z_{n}, \\
\mathrm{D}_{2}^{\theta} & =\frac{3}{64} \frac{A_{1,2}^{2}}{\mu^{2}} \sum_{n=3}^{6} \bar{K}_{n}+\frac{3}{128} A_{2,4} \sum_{n=7}^{10} \bar{K}_{n} \\
& +3 B_{2} \bar{K}_{11}+3 A_{2,2}^{S R P} \mathrm{~K}_{0 L}^{s}, \\
\mathrm{D}_{3}^{\theta} & =B_{3,2} \sum_{n=12}^{13} \bar{K}_{n}+\frac{A_{1,2}}{\mu^{2}} B_{2} \sum_{n=14}^{15} \bar{K}_{n} \\
& +\frac{27\left(A_{2,2}^{S R P}\right)^{2}}{8 A_{12}} \mathrm{~K}_{0 L}^{s s}+\frac{A_{11} A_{2,2}^{S R P}}{4 \mu^{2}}\left(\mathrm{~K}_{1 L}^{g s}+\mathrm{K}_{1 G}^{g s}\right) \\
& +\frac{27 A_{11}\left(A_{2,2}^{S R P}\right)^{2}}{64 A_{12}^{2}}\left(\mathrm{~K}_{3 L}^{g s}+\mathrm{K}_{4 G}^{g s}\right)
\end{aligned}
$$$$
\mathrm{D}_{3}^{h}=B_{3,2} Z_{7}+\frac{A_{1,2}}{\mu^{2}} B_{2} Z_{8}-\frac{27 C_{\varepsilon} L^{2}\left(A_{2,2}^{S R P}\right)^{2}}{16 A_{12}}
$$$$
+\frac{3 A_{11} A_{2,2}^{S R P}}{8 \mu^{2}} \mathrm{~K}_{1 H}^{g s}+\frac{27 A_{11} A_{24}^{2}}{16 A_{12}^{2}} \mathrm{~K}_{2 H}^{g s},
$$

where the nonvanishing coefficients $Z_{i}, i=1, \ldots . .8$ and $\bar{K}_{j}, j=1, \ldots, 15$ are given by Abd El-Salam, et. al. (2006). We will write only the newly computed coefficients which account for the contributions due to the direct solar radiation pressure and theralatevistic corrections

$$
\begin{aligned}
& Z_{7}=6 C\left(-\frac{3}{4} \eta_{3,6}+\frac{5}{8} \eta_{5,4}\right), \\
& Z_{8}=6 C\left(-\frac{45}{4} \eta_{3,6}-\frac{27}{4} \eta_{4,5}+9 \eta_{5,4}\right), \\
& \bar{K}_{11}=\left(3 \eta_{4,1}-\frac{5}{2} \eta_{5,0}+\eta_{3,2}\right), \\
& \bar{K}_{12}=\left(\frac{25}{8} \eta_{6,3}+\frac{15}{8} \eta_{5,4}-\frac{9}{4} \eta_{4,5}-\frac{15}{4} \eta_{3,6}\right), \\
& \bar{K}_{13}=3 C^{2}\left(\frac{21}{4} \eta_{3,6}-\frac{25}{8} \eta_{5,4}+\frac{9}{4} \eta_{4,5}-\frac{25}{8} \eta_{6,3}\right),
\end{aligned}
$$

Vol. 5 No. 9 (Sep. 2012)

ISSN: 0974- 6846

$$
\begin{aligned}
& \bar{K}_{14}=\left(45 \eta_{6,3}-\frac{243}{4} \eta_{4,5}-\frac{225}{4} \eta_{3,6}\right), \\
& \bar{K}_{15}=3 C^{2}\left(-45 \eta_{6,3}-18 \eta_{5,4}+\frac{297}{4} \eta_{4,5}+\frac{315}{4} \eta_{3,6}\right) \\
& \mathrm{K}_{0 L}^{s s}=-C_{\varepsilon} \eta_{-1-1}, \quad \mathrm{~K}_{0 L}^{s}=\left(\eta_{0-1}-\eta_{-1,0}\right) \text {, } \\
& \mathrm{K}_{1 L}^{g s}=-\left(\eta_{0-2}-3 H^{2}\right)\left(\eta_{-2,0}-\eta_{0-2}\right)^{-2}\left[5 \eta_{5,-6}\right. \\
& +3 \eta_{4,-5}-10 \eta_{3,-4}-66 \eta_{2,-3}+36 \eta_{1,-2} \\
& +465 \eta_{-1,0}-723 \eta_{1,-2}-606 \eta_{2,-1}+1419 \eta_{3,-2} \\
& \left.+326 \eta_{4,-3}-717 \eta_{6,-5}-351 \eta_{7,-6}+288 \eta_{9,-8}\right] \\
& \mathrm{K}_{1 G}^{g s}=\frac{1}{2}\left(\eta_{-2,0}-\eta_{0-2}\right)^{-2}\left[20 \eta_{-6,5}+18 \eta_{-5,4}\right. \\
& +21 \eta_{-4,3}+84 \eta_{-3,2}-102 \eta_{-2,1}-600 \eta_{0,-1} \\
& -564 \eta_{6,-7}+432 \eta_{8,-9}-6 H^{2}\left(15 \eta_{-6,7}+15 \eta_{-5,6}\right. \\
& +3 \eta_{-4,5}+150 \eta_{-3,4}-138 \eta_{-2,3}-456 \eta_{-1,2} \\
& +123 \eta_{0,1}+426 \eta_{1,0}-9 \eta_{2,-1}+87 \eta_{3,-2} \\
& \left.\left.-6 \eta_{4,-3}-213 \eta_{6,-5}+144 \eta_{8,-7}\right)\right] \\
& \mathrm{K}_{3 G}^{g s}=-\left(1+C_{\varepsilon}^{2}+2 S_{\varepsilon}^{2}\right)\left(\eta_{2,3}+2 \eta_{0,5}\right)-2 H^{2} \\
& \times\left[\left(1+C_{\varepsilon}^{2}-12 S_{\varepsilon}^{2}\right) \eta_{2,5}-3\left(1+C_{\varepsilon}^{2}+6 S_{\varepsilon}^{2}\right) \eta_{0,7}\right] \\
& +5 H^{4}\left(1+C_{\varepsilon}^{2}-2 S_{\varepsilon}^{2}\right)\left(3 \eta_{2,7}+4 \eta_{0,9}\right) \\
& \mathrm{K}_{4 G}^{g s}=\left(1+C_{\varepsilon}^{2}+2 S_{\varepsilon}^{2}\right)\left(10 \eta_{-1,6}-3 \eta_{1,4}\right)-42 H^{2} \\
& \times\left(1+C_{\varepsilon}^{2}+6 S_{\varepsilon}^{2}\right) \eta_{-1,8}+\left(1+C_{\varepsilon}^{2}-12 S_{\varepsilon}^{2}\right) \\
& \times 10 H^{2} \eta_{1,6}-75 H^{4}\left(1+C_{\varepsilon}^{2}-2 S_{\varepsilon}^{2}\right) \eta_{-1,10}
\end{aligned}
$$

$$
\begin{aligned}
\mathrm{K}_{1 H}^{g s}= & \left(\eta_{-2,0}-\eta_{0-2}\right)^{-2} H\left[10 \eta_{-4,6}+12 \eta_{-3,5}+51 \eta_{-2,4}\right. \\
& +228 \eta_{-1,3}-123 \eta_{0,2}-684 \eta_{1,1}+723 \eta_{2,0} \\
& \left.+348 \eta_{3,-1}-705 \eta_{4,-2}+6 \eta_{6,-4}+144 \eta_{8,-6}\right] \\
\mathrm{K}_{2 H}^{g s}= & 3 H\left(1+C_{\varepsilon}^{2}+6 S_{\varepsilon}^{2}\right) \eta_{-1,7}+H\left(1+C_{\varepsilon}^{2}-12 S_{\varepsilon}^{2}\right) \eta_{1,5} \\
& +5 H^{3}\left(1+C_{\varepsilon}^{2}-2 S_{\varepsilon}^{2}\right)\left(-3 \eta_{1,7}+4 \eta_{-1,9}\right)
\end{aligned}
$$

The second order constraints

In order to prevent two neighboring orbits from drifting apart, the average secular growth needs to be equal. To keep the satellites from drifting apart over time, it would be desirable to match all three rates Therefore 


$$
\left.\begin{array}{l}
\dot{\theta}_{i}=\dot{l}+\dot{g}=\dot{\theta}_{j} \\
\dot{h}_{i}=\dot{h}_{j}, \quad \forall i \neq j
\end{array}\right\}
$$

where $\theta$ is the mean argument of latitude. Denoting the reference mean orbital elements with the subscript " 0 " the Taylor expansion for the drift rates $\left\{\dot{\Xi}_{i}, \Xi \equiv \theta, h\right\}$ of a neighboring orbit about the reference orbit element, retaining the terms up the second order derivatives, can be written as

$$
\begin{aligned}
\dot{\Xi}_{i}= & \left(\dot{\Xi}_{i}\right)_{0}+\delta L\left(\dot{\Xi}_{i, L}\right)_{0}+\delta G\left(\dot{\Xi}_{i, G}\right)_{0} \\
& +\delta H\left(\dot{\Xi}_{i, H}\right)+\delta K\left(\dot{\Xi}_{i, K}\right)_{0} \\
& +\frac{1}{2 !}\left\{(\delta L)^{2}\left(\dot{\Xi}_{i, L L}\right)_{0}+(\delta G)^{2}\left(\dot{\Xi}_{i, G G}\right)_{0}\right. \\
& +(\delta H)^{2}\left(\dot{\Xi}_{i, H H}\right)_{0}+(\delta K)^{2}\left(\dot{\Xi}_{i, K K}\right)_{0} \\
& +2(\delta L)(\delta G)\left(\dot{\Xi}_{i, L G}\right)_{0} \\
& +2(\delta G)(\delta H)\left(\dot{\Xi}_{i, G H}\right)_{0} \\
& \left.+2(\delta H)(\delta L)\left(\dot{\Xi}_{i, H L}\right)_{0}\right\} \\
& +2(\delta K)(\delta L)\left(\dot{\Xi}_{i, K L}\right)_{0} \\
& +2(\delta K)(\delta G)\left(\dot{\Xi}_{i, K G}\right)_{0} \\
& \left.+2(\delta K)(\delta H)\left(\dot{\Xi}_{i, K H}\right)_{0}\right\}
\end{aligned}
$$

Where $\left(\dot{\Xi}_{i, x y}\right)_{0}=\left.\frac{\partial^{2} \dot{\Xi}_{i}}{\partial x \partial y}\right|_{x=x_{0}, y=y_{0}}=\sum_{n=0}^{3} \frac{J_{2}^{n}}{n !} \mathrm{D}_{n, x y}^{\Xi_{i}}$ are the required partial derivatives with $x, y \equiv L, G, H, K$, evaluated at the mean orbital element of the reference orbit $L_{o}, G_{o}, H_{o}, K_{o}$. The nonvanishing coefficients $\mathrm{D}^{\Xi_{i}}\left(\equiv \mathrm{D}^{\theta}, \mathrm{D}^{h}\right)$ which include the relativistic and the direct solar radiation pressure contributions to the theory of invariant relative orbits are
Vol. 5 No. 9 (Sep. 2012)

ISSN: 0974- 6846

computed in the appendix A. Let $\eta=G / L$, therefore we can write

$$
\left.\begin{array}{l}
\delta G=\eta(\delta L)+L(\delta \eta), \\
\delta H=\eta C(\delta L)+L C(\delta \eta)-L \eta S(\delta I)
\end{array}\right\}
$$

It worth to note here that $\delta L, \delta G$, and $\delta H$ are independent. Therefore equation (8) can be rewritten in terms of $\delta L, \delta \eta$, and $\delta I$ in the form

$$
\begin{aligned}
& \delta \dot{\Xi}=\left[\left(\dot{\Xi}_{i, L}\right)_{0}+\eta\left(\dot{\Xi}_{i, G}\right)_{0}+\eta C\left(\dot{\Xi}_{i, H}\right)_{0}\right] \delta L \\
& +\left[L\left(\dot{\Xi}_{i, G}\right)_{0}+L C\left(\dot{\Xi}_{i, H}\right)_{0}\right] \delta \eta \\
& +\left[\operatorname{L\eta } S\left(\dot{\Xi}_{i, H}\right)_{0}\right] \delta I+\left[\frac{1}{2}\left(\dot{\Xi}_{i, L L}\right)_{0}\right. \\
& +\frac{1}{2} \eta^{2}\left(\dot{\Xi}_{i, G G}\right)_{0}+\frac{1}{2} \eta^{2} C^{2}\left(\dot{\Xi}_{i, H H}\right)_{0} \\
& +\eta\left(\dot{\Xi}_{i, L G}\right)_{0}+\eta^{2} C\left(\dot{\Xi}_{i, G H}\right)_{0} \\
& \left.+\eta C\left(\dot{\Xi}_{i, H L}\right)_{0}\right](\delta L)^{2}+\left[\frac{1}{2} L^{2}\left(\dot{\Xi}_{i, G G}\right)_{0}\right. \\
& \left.+\frac{1}{2} L^{2} C^{2}\left(\dot{\Xi}_{i, H H}\right)_{0}+\frac{1}{2} L^{2} C\left(\dot{\Xi}_{i, G H}\right)_{0}\right] \\
& \times(\delta \eta)^{2}+\left[\frac{1}{2} L^{2} \eta^{2} S^{2}\left(\dot{\Xi}_{i, H H}\right)_{0}\right](\delta I)^{2} \\
& +\left[\operatorname{L\eta }\left(\dot{\Xi}_{i, G G}\right)_{0}+L \eta C^{2}\left(\dot{\Xi}_{i, H H}\right)_{0}\right. \\
& +L\left(\dot{\Xi}_{i, L G}\right)_{0}+2 L \eta C\left(\dot{\Xi}_{i, G H}\right)_{0} \\
& \left.+L C\left(\dot{\Xi}_{i, H L}\right)_{0}\right](\delta L)(\delta \eta)-\left[L^{2} \eta C\right. \\
& \left.\times\left(\dot{\Xi}_{i, H H}\right)_{0}+L^{2} \eta C\left(\dot{\Xi}_{i, G H}\right)_{0}\right](\delta \eta)(\delta I) \\
& -\left[L C \eta\left(\dot{\Xi}_{i, H H}\right)_{0}+L \eta^{2} S\left(\dot{\Xi}_{i, G H}\right)_{0}\right. \\
& \left.+L \eta S\left(\dot{\Xi}_{i, L G}\right)_{0}\right](\delta I)(\delta L)
\end{aligned}
$$

(9) where $\delta \dot{\Xi}\left(\equiv \dot{\theta}=\dot{\theta}_{i}-\dot{\theta}_{0}, \delta \dot{h}=\dot{h}_{i}-\dot{h}_{0}\right)$ are the difference between the drift rate of the argument of mean latitude of the reference orbit and that of the neighboring orbit, and the difference between the drift rate of the ascending node of the reference orbit and that of the neighboring orbit respectively. The conditions satisfying 
the invariance property for the relative orbits are $\delta \dot{\Xi}=0$. Substituting the included derivatives, in the appendix $A$, into equations (9) yields

$$
\begin{aligned}
\delta \dot{\Xi} & =0=\mathrm{A}_{L}^{\Xi} \delta L+\mathrm{A}_{\eta}^{\Xi} \delta \eta+\mathrm{A}_{I}^{\Xi} \delta I+\mathrm{A}_{L L}^{\Xi}(\delta L)^{2} \\
& +\mathrm{A}_{\eta \eta}^{\Xi}(\delta \eta)^{2}+\mathrm{A}_{I I}^{\Xi}(\delta I)^{2}+\mathrm{A}_{L \eta}^{\Xi}(\delta L)(\delta \eta) \\
& +\mathrm{A}_{\eta I}^{\Xi}(\delta \eta)(\delta I)+\mathrm{A}_{I L}^{\Xi}(\delta I)(\delta L)
\end{aligned}
$$

where

$$
\begin{array}{lll}
\mathrm{A}_{L}^{\theta}=\sum_{n=1}^{3} J_{2}^{n} \mathrm{I}_{n}^{\theta}, & \mathrm{A}_{\eta}^{\theta}=\sum_{n=1}^{3} J_{2}^{n} \mathrm{~K}_{n}^{\theta}, & \mathrm{A}_{I}^{\theta}=\sum_{n=1}^{3} J_{2}^{n} \mathrm{~L}_{n}^{\theta}, \\
\mathrm{A}_{I I}^{\theta}=\sum_{n=1}^{3} J_{2}^{n} \mathrm{P}_{n}^{\theta}, & \mathrm{A}_{L \eta}^{\theta}=\sum_{n=1}^{3} J_{2}^{n} \mathrm{Q}_{n}^{\theta}, & \mathrm{A}_{I L}^{\theta}=\sum_{n=1}^{3} J_{2}^{n} \mathrm{~S}_{n}^{\theta}, \\
\mathrm{A}_{I}^{h}=\sum_{n=1}^{3} J_{2}^{n} \mathrm{~L}_{n}^{h}, & \mathrm{~A}_{\eta}^{h}=\sum_{n=1}^{3} J_{2}^{n} \mathrm{~K}_{n}^{h}, & \mathrm{~A}_{L L}^{\theta}=\sum_{n=1}^{3} J_{2}^{n} \mathrm{M}_{n}^{\theta}, \\
\mathrm{A}_{L L}^{h}=\sum_{n=1}^{3} J_{2}^{n} \mathrm{M}_{n}^{h}, & \mathrm{~A}_{\eta \eta}^{h}=\sum_{n=1}^{3} J_{2}^{n} \mathrm{~N}_{n}^{h}, & \mathrm{~A}_{I I}^{h}=\sum_{n=1}^{3} J_{2}^{n} \mathrm{P}_{n}^{h}, \\
\mathrm{~A}_{\eta \eta}^{\theta}=\sum_{n=1}^{3} J_{2}^{n} \mathrm{~N}_{n}^{\theta}, & \mathrm{A}_{L}^{h}=\sum_{n=1}^{3} J_{2}^{n} \mathrm{I}_{n}^{h}, & \mathrm{~A}_{\eta I}^{\theta}=\sum_{n=1}^{3} J_{2}^{n} \mathrm{R}_{n}^{\theta}, \\
\mathrm{A}_{L \eta}^{h}=\sum_{n=1}^{3} J_{2}^{n} \mathrm{Q}_{n}^{h}, & \mathrm{~A}_{\eta I}^{h}=\sum_{n=1}^{3} J_{2}^{n} \mathrm{R}_{n}^{h}, & \mathrm{~A}_{I L}^{h}=\sum_{n=1}^{3} J_{2}^{n} \mathrm{~S}_{n}^{h}
\end{array}
$$

where the functional forms of the included coefficients $\mathrm{S}$, I , K , L , P , M , N , R , Q are obtained previously by the Abd El-Salam et al. (2012) equation (22) there.

\section{Special case: First order constraints}

Neglecting the terms higher than the first order, and also neglecting the squared and the mixed differences of $\delta L, \delta \eta$, and $\delta I$ in equations (10), we get

$$
\begin{aligned}
& \mathrm{I}_{0}^{\theta} \delta L+J_{2}\left(\mathrm{I}_{1}^{\theta} \delta L+\mathrm{K}_{1}^{\theta} \delta \eta+\mathrm{L}_{1}^{\theta} \delta I\right)=0 \\
& J_{2}\left(\mathrm{I}_{1}^{h} \delta L+\mathrm{K}_{1}^{h} \delta \eta+\mathrm{L}_{1}^{h} \delta I\right)=0
\end{aligned}
$$

Considering $\delta L$ of order $\mathrm{O}\left(J_{2}\right)$ as suggested by Schaub and Alfriend [4], terms factored by $J_{2} \delta L$ can be neglected, we get

$$
\left.\begin{array}{l}
\mathrm{I}_{0}^{\theta} \delta L+J_{2}\left(\mathrm{~K}_{1}^{\theta} \delta \eta+\mathrm{L}_{1}^{\theta} \delta I\right)=0 \\
J_{2}\left(\mathrm{~K}_{1}^{h} \delta \eta+\mathrm{L}_{1}^{h} \delta I\right)=0
\end{array}\right\}
$$

rearranging the terms, equation (12) can be written as

$$
\delta \eta=-\left(\mathrm{L}_{1}^{h} / \mathrm{K}_{1}^{h}\right) \delta I
$$

Solving equation (13) for $\delta I$ and substitute into the $1^{\text {st }}$ equation of (12), we obtain

$$
\mathrm{I}_{0}^{\theta} \delta L+J_{2}\left(\mathrm{~K}_{1}^{\theta} \delta \eta-\mathrm{L}_{1}^{\theta}\left(\mathrm{K}_{1}^{h} / \mathrm{L}_{1}^{h}\right) \delta \eta\right)=0
$$

rearranging the terms, equation (14) can be written in the form

$\delta L=-\left(J_{2} / \mathrm{I}_{0}^{\theta}\right)\left[\mathrm{K}_{1}^{\theta}-\mathrm{L}_{1}^{\theta}\left(\mathrm{K}_{1}^{h} / \mathrm{L}_{1}^{h}\right)\right] \delta \eta$
Vol. 5 No. 9 (Sep. 2012)

ISSN: 0974- 6846

using the coefficients $\mathrm{L}_{1}^{h}, \mathrm{~K}_{1}^{h}, \mathrm{I}_{0}^{\theta}, \mathrm{K}_{1}^{\theta}, \mathrm{L}_{1}^{\theta}$, defined previously, with $\eta_{i, j}=L^{-i-j} \eta^{-j}$, assuming the distances are measured in Earth radius and the time is normalized by the mean motion of a satellite at one Earth radius, $R_{\oplus}=1$ and $\mu=1$, equations (13) and (15) give

$$
\left.\begin{array}{l}
\delta \eta=-\frac{\eta}{4}(\tan I) \delta I, \\
\delta L=\frac{J_{2}}{4 L^{4} \eta^{5}}\left[(3 \eta+4)\left(5 C^{2}+1\right)\right] L \delta \eta
\end{array}\right\}
$$

which are the same first order constraints obtained by Schaub and Alfriend (2002).

Solution of the second order constraints

Equations (9) are two simultaneous nonlinear algebraic equations in three unknowns, namely $\delta L, \delta \eta$ , $\delta I$. When one of these three unknowns is assumed known (say $\delta I$ ), these two equations can be solved as follows. Rewriting equations (9) as

$$
\begin{aligned}
0 & =\mathrm{A}_{L L}^{\Xi_{i}}(\delta L)^{2}+\mathrm{A}_{L \eta}^{\Xi_{i}}(\delta L)(\delta \eta)+\mathrm{A}_{\eta \eta}^{\Xi_{i}}(\delta \eta)^{2}+\mathrm{A}_{I}^{\Xi_{i}} \delta I \\
& +\mathrm{A}_{I I}^{\Xi_{i}}(\delta I)^{2}+\left[\mathrm{A}_{L}^{\Xi_{i}}+\mathrm{A}_{I L}^{\Xi_{i}}(\delta I)\right](\delta L) \\
& +\left[\mathrm{A}_{\eta}^{\Xi_{i}}+\mathrm{A}_{\eta I}^{\Xi_{i}}(\delta I)\right](\delta \eta)
\end{aligned}
$$

Multiplying the first equation of (17) by $\mathrm{A}_{L L}^{h}$ and the second equation (17) by $\mathrm{A}_{L L}^{\theta}$ and then subtracting yields $c_{1}(\delta L)(\delta \eta)+c_{2}(\delta \eta)^{2}+c_{3}(\delta L)+c_{4}(\delta \eta)+c_{5}=0(18)$ where

$$
\begin{aligned}
& c_{1}=\left(\mathrm{A}_{L \eta}^{\theta}\right)\left(\mathrm{A}_{L L}^{h}\right)-\left(\mathrm{A}_{L L}^{\theta}\right)\left(\mathrm{A}_{L \eta}^{h}\right) \\
& c_{2}=\left(\mathrm{A}_{\eta \eta}^{\theta}\right)\left(\mathrm{A}_{L L}^{h}\right)-\left(\mathrm{A}_{L L}^{\theta}\right)\left(\mathrm{A}_{\eta \eta}^{h}\right) \\
& c_{3}=\left[\mathrm{A}_{L}^{\theta}+\mathrm{A}_{I L}^{\theta}(\delta I)\right]\left(\mathrm{A}_{L L}^{h}\right)-\left(\mathrm{A}_{L L}^{\theta}\right)\left[\mathrm{A}_{L}^{h}+\mathrm{A}_{I L}^{h}(\delta I)\right] \\
& c_{4}= {\left[\mathrm{A}_{\eta}^{\theta}+\mathrm{A}_{\eta I}^{\theta}(\delta I)\right]\left(\mathrm{A}_{L L}^{h}\right)-\left(\mathrm{A}_{L L}^{\theta}\right)\left[\mathrm{A}_{\eta}^{h}+\mathrm{A}_{\eta I}^{h}(\delta I)\right] } \\
& c_{5}=\left[\mathrm{A}_{I}^{\theta} \delta I+\mathrm{A}_{I I}^{\theta}(\delta I)^{2}\right]\left(\mathrm{A}_{L L}^{h}\right) \\
&-\left(\mathrm{A}_{L L}^{\theta}\right)\left[\mathrm{A}_{I}^{h} \delta I+\mathrm{A}_{I I}^{h}(\delta I)^{2}\right]
\end{aligned}
$$

From equation (18), we can get

$$
(\delta L)=-\frac{c_{2}(\delta \eta)^{2}+c_{4}(\delta \eta)+c_{5}}{c_{1}(\delta \eta)+c_{3}}
$$

Substituting equation (19) into the first equation of (17) yields an algebraic equation of fourth degree in $\delta \eta$ only in the form

$d_{1}(\delta \eta)^{4}+d_{2}(\delta \eta)^{3}+d_{3}(\delta \eta)^{2}+d_{4} \delta \eta+d_{5}=0$

where

$d_{1}=c_{2}^{2}\left(\mathrm{~A}_{L L}^{\theta}\right)-c_{2} c_{1}\left(\mathrm{~A}_{L \eta}^{\theta}\right)+c_{1}^{2}\left(\mathrm{~A}_{\eta \eta}^{\theta}\right)$ 


$$
\begin{aligned}
d_{2} & =2 c_{2} c_{4}\left(\mathrm{~A}_{L L}^{\theta}\right)-\left[c_{2} c_{3}+c_{4} c_{1}\right]\left(\mathrm{A}_{L \eta}^{\theta}\right) \\
& -c_{2} c_{1}\left[\mathrm{~A}_{L}^{\theta}+\mathrm{A}_{I L}^{\theta}(\delta I)\right]+c_{1}^{2}\left[\mathrm{~A}_{\eta}^{\theta}\right. \\
& \left.+\mathrm{A}_{\eta I}^{\theta}(\delta I)\right]+2 c_{1} c_{3}\left(\mathrm{~A}_{\eta \eta}^{\theta}\right) \\
d_{3}= & {\left[c_{4}^{2}+2 c_{2} c_{5}\right]\left(\mathrm{A}_{L L}^{\theta}\right)-\left[c_{4} c_{3}+c_{5} c_{1}\right]\left(\mathrm{A}_{L \eta}^{\theta}\right) } \\
& +c_{3}^{2}\left(\mathrm{~A}_{\eta \eta}^{\theta}\right)-\left[c_{2} c_{3}+c_{4} c_{1}\right]\left[\mathrm{A}_{L}^{\theta}+\mathrm{A}_{I L}^{\theta}(\delta I)\right] \\
& +2 c_{1} c_{3}\left[\mathrm{~A}_{\eta}^{\theta}+\mathrm{A}_{\eta I}^{\theta}(\delta I)\right]+c_{1}^{2}\left[\mathrm{~A}_{I}^{\theta} \delta I+\mathrm{A}_{I I}^{\theta}(\delta I)^{2}\right] \\
d_{4} & =2 c_{4} c_{5}\left(\mathrm{~A}_{L L}^{\theta}\right)-c_{5} c_{3}\left(\mathrm{~A}_{L \eta}^{\theta}\right)-\left[c_{4} c_{3}+c_{5} c_{1}\right] \\
& \times\left[\mathrm{A}_{L}^{\theta}+\mathrm{A}_{I L}^{\theta}(\delta I)\right]+c_{3}^{2}\left[\mathrm{~A}_{\eta}^{\theta}+\mathrm{A}_{\eta I}^{\theta}(\delta I)\right] \\
d_{5} & =c_{5}^{2}\left(\mathrm{~A}_{L L}^{\theta}\right)-c_{5} c_{3}\left[\mathrm{~A}_{L}^{\theta}+\mathrm{A}_{I L}^{\theta}(\delta I)\right] \\
& +c_{3}^{2}\left[\mathrm{~A}_{I}^{\theta} \delta I+\mathrm{A}_{I I}^{\theta}(\delta I)^{2}\right] \\
& +2 c_{1} c_{3}\left[\mathrm{~A}_{I}^{\theta} \delta I+\mathrm{A}_{I I}^{\theta}(\delta I)^{2}\right]
\end{aligned}
$$

\section{Solution of the quartic equation (20)}

The roots of the quartic equation (20) can be written as

$$
\begin{aligned}
& (\delta \eta)_{1,2}=\mathrm{A}-\mathrm{B} \mp \mathrm{C}, \\
& (\delta \eta)_{3,4}=\mathrm{A}+\mathrm{B} \mp \mathrm{D}
\end{aligned}
$$

where

$$
\begin{aligned}
& \mathrm{A}=-\frac{d_{2}}{4 d_{1}}, \quad \mathrm{~B}=\frac{1}{2} \sqrt{\mathrm{T}+\mathrm{C}+\mathrm{F}}, \\
& \mathrm{C}_{1,2}=\frac{1}{2} \sqrt{2 \mathrm{~T}-\mathrm{C}-\mathrm{F} \mp \mathrm{E}}
\end{aligned}
$$

with

$$
\begin{aligned}
\mathrm{T} & =\frac{d_{2}^{2}}{4 d_{1}^{2}}-\frac{2 d_{3}}{3 d_{1}}, \\
\mathrm{U} & =\left(d_{3}^{2}-3 d_{2} d_{4}+12 d_{1} d_{5}\right), \\
\mathrm{C} & =\sqrt[3]{2} \mathrm{U} / 3 d_{1} \mathrm{Z}, \\
\mathrm{V} & =2 d_{3}^{3}-9 d_{2} d_{3} d_{4}+27 d_{1} d_{4}^{2} \\
+ & 27 d_{2}^{2} d_{5}-72 d_{1} d_{3} d_{5} \\
\mathrm{Z} & =\left(\mathrm{V}+\sqrt{-4 \mathrm{U}^{3}+\mathrm{V}^{2}}\right)^{\frac{1}{3}} \\
\mathrm{~F} & =\mathrm{Z} / 3 \sqrt[3]{2} d_{1}, \\
\mathrm{G}= & -\left(d_{2} / d_{1}\right)^{3}+4 d_{2} d_{3} / d_{1}^{2}-8 d_{4} / d_{1}, \\
\mathrm{E} & =\mathrm{G} / 8 \mathrm{~B}
\end{aligned}
$$

Substituting the four roots $\delta \eta \dot{ } \eta^{\prime}$ into equation (20) yields the four constraints $\delta L^{\prime} s$ that guarantee the invariance of the relative motion of certain satellite constellation
Vol. 5 No. 9 (Sep. 2012)

ISSN: 0974- 6846

$$
\begin{aligned}
&(\delta L)_{3,4}=-\frac{1}{c_{1}\left(\mathrm{~A}+\mathrm{B}-\mathrm{C}_{2}\right)+c_{3}} \\
& \times\left[c_{2}\left(\mathrm{G}+2 \mathrm{AB} \mp 2 \mathrm{BC}_{2} \mp 2 \mathrm{AD}\right)\right. \\
&\left.+c_{4}\left(\mathrm{~A}+\mathrm{B}-\mathrm{C}_{2}\right)+c_{5}\right] \\
& \mathrm{F} \equiv \mathrm{A}^{2}+\mathrm{B}^{2}+\mathrm{C}_{1}^{2}, \quad \mathrm{G} \equiv \mathrm{A}^{2}+\mathrm{B}^{2}+\mathrm{C}_{2}^{2}
\end{aligned}
$$

\section{Computational algorithm}

Given Data

1. $a:$ The semi -major axis of the master orbit, $e:$ The eccentricity of the master orbit, $I$ : The inclination of the master orbit, $\delta I$ : The desired difference in inclination between the master orbit and the neighboring orbit, $\mu$ : Earth's gravitational constant, $R_{\oplus}$ : The mean equatorial radius, $J_{2}$ : The second zonal harmonic, $J_{4}$ : The fourth zonal harmonic, $c_{l}$ : The velocity of light.

2. Compute the following expressions $C, S, C_{\varepsilon}, S_{\varepsilon}, L, \eta, \eta_{i, j}$,

$A_{1,2}, A_{2,4}, B_{2}, B_{3,2}, A_{2,2}^{S R P}$

3. Compute the following coefficients D's (see Appendix A).

4. Compute the following coefficients $\mathrm{S}, \mathrm{I}, \mathrm{K}, \mathrm{L}, \mathrm{P}$ , M , N , R , see Abd El-Salam et al. (2006) equation (22) there.

5. Compute the following coefficients $\mathrm{A}^{\prime} s$, see equations (10).

6. Solving equation (20) either numerically or analytically, as given by the expressions (21) and (22) yields the four roots for $\delta \eta$.

7. Compute the four constraints $\delta L^{\prime} s$ that guarantee the invariance of the relative motion of certain satellite constellation as given by the expressions (21) and (22) Conclusion

Using the oblate Earth model, truncating its potential series at $J_{4}$, plus the relativistic corrections and the direct solar radiation pressure to the equations of motion, eight second order conditions between the differences in momenta elements (semi-major axis $a$, eccentricity $e$, inclination $I$ ) are obtained. These conditions guarantee that the drift rates of two neighboring orbits are equal on the average. The resulting orbits require less control and 
maintenance fuel. The two first order conditions obtained by Schaub and Alfriend (2001) are derived as a special case of the presented work. Finally a computational algorithm is presented.

Acknowledgment

The authors are deeply indebted and thankful to the deanship of the scientific research for his helpful and for distinct team work of employees at Taibah University, AlMadinah Al-Munawwarah, K. S. A. This research work was supported by a grant No (164 / 1432) from the deanship of the scientific research at Taibah university, Al-Madinah Al-Munawwarah, K.S.A.

\section{References}

1. Abd El-Salam FA (2012) Relativistic corrections to the theory of the invariant relative orbits of satellite flying formations. Intl. J. Nonlinear Sci. (IJNS). 13(2), 131-141.

2. Abd El-Salam FA, Ahmed MKM and Radwan M (2005) The post-newtonian exects in the critical inclination problem in arti.cial satellite theory. Appli. Maths \& Comput., 161, 813-823.

3. Abd El-Salam FA, El-Tohamy IA, Ahmed MK, Rahoma WA and Rassem MA (2006) Invariant relative orbits for satellite constellations: A second order theory. Appli. Maths. \& Comput. 181(1), 6 - 20.

4. Alfriend KT, Vadali SR and Schaub H (2001) Formation flying satellites: Control by an astrodynamist. Celest. Mech. 81, pp: 57.

5. Alfriend KT, Gim DW and and Vadali SR (2002) The characterization of formation flying satellite relative motion orbit. AASO. 2, pp: 143.

6. Easton RL and Brescia R (1969) Continuously visible satellite constellations. Naval Res. Lab. Report . pp: 6896.

7. Hughes SP (1999) Formation flying performance measures for earth-pointing missions. MSc Thesis, Blacksburg, Virginia.

8. Kamel AA (1969) Celestial mechanics 1, 190.

9. EL-Saftawy M, Ahmed MKM and Helali YE (1998) Astrophys. Space Sci. 259, 141.

10. Schaub H and Alfriend KT (2001) J2 invariant relative orbits for spacecraft formations. Celest. Mech. 79, pp: 77.

\section{Appendix A}

The required coefficients in equation (8) are

$$
\begin{aligned}
\mathrm{D}_{2, L}^{\theta}= & \frac{3}{64} \frac{A_{1,2}^{2}}{\mu^{2}}\left[\eta_{5,7}\left(-36+648 C^{2}-1860 C^{4}\right)\right. \\
& +\eta_{6,6}\left(-205+1110 C^{2}-930 C^{4}\right) \\
& +\eta_{7,5}\left(-150+540 C^{2}-150 C^{4}\right) \\
& \left.+\eta_{4,8}\left(105-270 C^{2}-1155 C^{4}\right)\right]+\frac{3}{128} A_{2,4} \\
& \times\left[\eta_{5,7}\left(180-1800 C^{2}+540 C^{2}-150 C^{4}\right)\right.
\end{aligned}
$$

Vol. 5 No. 9 (Sep. 2012)

ISSN: 0974- 6846

$$
\begin{aligned}
& \left.+\eta_{4,8}\left(105-270 C^{2}-1155 C^{4}\right)\right]+\frac{3}{128} A_{2,4} \\
& \times\left[\eta_{5,7}\left(180-1800 C^{2}+2100 C^{4}\right)\right. \\
& -\eta_{7,5}\left(270-2700 C^{2}+3150 C^{4}\right) \\
& +\eta_{4,8}\left(315-4050 C^{2}+5775 C^{4}\right) \\
& \left.-\eta_{6,6}\left(225-3150 C^{2}+4725 C^{4}\right)\right] \\
& -3 B_{2}\left(12 \eta_{5,1}-\frac{25}{2} \eta_{6,0}+3 \eta_{4,2}\right)-3 A_{2,2}^{S R P} \\
& \mathrm{D}_{3, L}^{\theta}=B_{3,2}\left\{\left(\frac{45}{4} \eta_{4,6}-\frac{75}{4} \eta_{7,3}-\frac{75}{8} \eta_{6,4}+9 \eta_{5,5}\right)\right. \\
& \left.+3 C^{2}\left(\frac{125}{8} \eta_{6,4}-\frac{63}{4} \eta_{4,6}-9 \eta_{5,5}+\frac{75}{4} \eta_{7,3}\right)\right\} \\
& +\frac{A_{1,2}}{\mu^{2}} B_{2}\left\{\left(243 \eta_{5,5}-270 \eta_{7,3}+\frac{675}{4} \eta_{4,6}\right)\right. \\
& \left.+3 C^{2}\left(270 \eta_{7,3}+90 \eta_{6,4}-297 \eta_{5,5}-\frac{945}{4} \eta_{4,6}\right)\right\} \\
& -\frac{27\left(A_{2,2}^{S R P}\right)^{2}}{8 A_{12}} C_{\varepsilon} \eta_{0,-1}+\frac{A_{11} A_{2,2}^{S R P}}{4 \mu^{2}}\left\{4\left(\eta_{0,-2}-3 H^{2}\right)\right. \\
& \times\left(\eta_{-2,0}-\eta_{0,-2}\right)^{-3}\left[-723+5 \eta_{-6,6}+3 \eta_{-5,5}\right. \\
& -10 \eta_{-4,4}-66 \eta_{-3,3}+36 \eta_{-2,2}+456 \eta_{-1,1}-606 \eta_{1,-1} \\
& +1419 \eta_{2,-2}+326 \eta_{3,-3}-717 \eta_{5,-5}-35 \eta_{6,-6} \\
& \left.+288 \eta_{8,-8}\right]-\left(\eta_{0,-2}-3 H^{2}\right)\left(\eta_{-2,0}-\eta_{0,-2}\right)^{-2} \\
& \times\left[723 \eta_{2,0}+25 \eta_{-4,6}+12 \eta_{-3,5}-30 \eta_{-2,4}-132 \eta_{-1,3}\right. \\
& +36 \eta_{0,2}+1212 \eta_{3,-2}-4257 \eta_{4,-2}-13044 \eta_{5,-3} \\
& \left.\left.+4302 \eta_{7,-5}+2457 \eta_{8,-6}-2592 \eta_{10,-8}\right]\right\} \\
& +27 A_{11}\left(A_{2,2}^{S R P} / 8 A_{12}\right)^{2}\left\{\left(1+C_{\varepsilon}^{2}+2 S_{\varepsilon}^{2}\right)\right. \\
& \times\left(-2 \eta_{3,3}+10 \eta_{0,6}+3 \eta_{2,4}\right)\left(4 \eta_{3,5}+10 \eta_{2,6}\right) \\
& +\left(1+C_{\varepsilon}^{2}-12 S_{\varepsilon}^{2}\right) H^{2}-42\left(1+C_{\varepsilon}^{2}+6 S_{\varepsilon}^{2}\right) H^{2} \eta_{0,8} \\
& \left.-\left(1+C_{\varepsilon}^{2}-2 S_{\varepsilon}^{2}\right) H^{4}\left(30 \eta_{3,7}+75 \eta_{0,10}\right)\right\} \\
& \mathrm{D}_{2, G}^{\theta}=\frac{3}{64} \frac{A_{1,2}^{2}}{\mu^{2}}\left[\eta_{4,8}\left(-63+1458 C^{2}-5115 C^{4}\right)\right. \\
& +\eta_{5,7}\left(-246+1776 C^{2}-1860 C^{4}\right)+\eta_{6,6}(-125
\end{aligned}
$$


Indian Journal of Science and Technology

$$
\begin{aligned}
& \left.\left.+630 C^{2}-225 C^{4}\right)+\eta_{3,9}\left(280-900 C^{2}-4620 C^{4}\right)\right] \\
& +\frac{3 A_{2,4}}{128}\left[\eta_{4,8}\left(315-4050 C^{2}+5775 C^{4}\right)\right. \\
& +\eta_{6,6}\left(-225+1125 C^{2}-4725 C^{4}\right) \\
& +\eta_{3,9}\left(840-13500 C^{2}+23100 C^{4}\right) \\
& \left.-\eta_{5,7}\left(270-5040 C^{2}+9450 C^{4}\right)\right] \\
& +3 B_{2}\left(-3 \eta_{4,2}-2 \eta_{3,3}\right)+3 A_{2,2}^{S R P} \\
& \mathrm{D}_{3, G}^{\Theta}=B_{3,2}\left\{\left(-\frac{75}{8} \eta_{6,4}-\frac{15}{2} \eta_{5,5}+\frac{45}{4} \eta_{4,6}+\frac{45}{2} \eta_{3,7}\right)\right. \\
& -3 B_{2}\left(3 \eta_{4,2}+2 \eta_{3,3}\right)+3 C^{2}\left(-42 \eta_{3,7}+\frac{75}{4} \eta_{5,5}\right. \\
& \left.\left.-\frac{63}{4} \eta_{4,6}+\frac{125}{4} \eta_{6,4}\right)\right\}+\frac{A_{1,2}}{\mu^{2}} B_{2}\left\{\left(-135 \eta_{6,4}\right.\right. \\
& \left.+\frac{1215}{4} \eta_{4,6}+\frac{675}{2} \eta_{3,7}\right)+\frac{A_{1,2}}{\mu^{2}} B_{2}\left\{\left(-135 \eta_{6,4}\right.\right. \\
& \left.+\frac{1215}{4} \eta_{4,6}+\frac{675}{2} \eta_{3,7}\right)+3 C^{2}\left(225 \eta_{6,4}+108 \eta_{5,5}\right. \\
& \left.\left.-\frac{2079}{4} \eta_{4,6}-\frac{2520}{4} \eta_{3,7}\right)\right\}-27\left(A_{2,2}^{2 S R P} / 8 A_{12}\right) C_{\varepsilon} \eta_{0,-1} \\
& +\frac{A_{11} A_{2,2}^{S R P}}{4 \mu^{2}}\left\{( \eta _ { 0 , - 2 } - 3 H ^ { 2 } ) ( \eta _ { - 2 , 0 } - \eta _ { 0 , - 2 } ) ^ { - 2 } \left[30 \eta_{-5,7}\right.\right. \\
& +15 \eta_{-4,6}-40 \eta_{-3,5}-198 \eta_{-2,4}+72 \eta_{-1,3}+456 \eta_{0,2} \\
& +606 \eta_{2,0}-2838 \eta_{3,-1}-9783 \eta_{4,-2}+3585 \eta_{6,-4} \\
& \left.+2106 \eta_{7,-5}-2304 \eta_{9,-7}\right]-2\left(\eta_{-2,0}-3 H^{2}\right) \\
& \times\left(\eta_{-2,0}-\eta_{0,-2}\right)^{-3}\left[5 \eta_{-5,5}+3 \eta_{-4,4}-10 \eta_{-3,3}\right. \\
& -66 \eta_{-2,2}+36 \eta_{-1,1}+456-723 \eta_{1,-1}-606 \eta_{2,-2} \\
& +1419 \eta_{3,-3}+3261 \eta_{4,-4}-717 \eta_{6,-6}-351 \eta_{7,-7} \\
& \left.+288 \eta_{9,-9}\right]+\frac{1}{2}\left(\eta_{-2,0}-\eta_{0,-2}\right)^{-2}\left[-100 \eta_{-6,6}\right. \\
& -72 \eta_{-5,5}+63 \eta_{-4,4}-168 \eta_{-3,3}+102 \eta_{-2,2}-600 \\
& -360 \eta_{1,-1}+4230 \eta_{2,-2}+696 \eta_{3,-3}-3615 \eta_{4,-4} \\
& -3948 \eta_{6,-6}+3888 \eta_{8,-8}-6 H^{2}\left(-105 \eta_{-6,8}\right. \\
& -90 \eta_{-5,7}-155 \eta_{-4,6}-600 \eta_{-3,5}+414 \eta_{-2,4} \\
& \left.\left.+174 \eta_{3,-1}-18 \eta_{4,-2}-1065 \eta_{6,-4}+1008 \eta_{-8,6}\right)\right]
\end{aligned}
$$

Vol. 5 No. 9 (Sep. 2012)

ISSN: 0974- 6846

$$
\begin{aligned}
& -600 \eta_{0,-1}-180 \eta_{1,-2}+1410 \eta_{2,-3}+174 \eta_{3,-4} \\
& -723 \eta_{4,-5}-564 \eta_{6,-5}+432 \eta_{8,-9}-6 H^{2}\left(15 \eta_{-6,7}\right. \\
& +15 \eta_{-5,6}+31 \eta_{-4,5}+150 \eta_{-3,4}-138 \eta_{-2,3}-456 \eta_{-1,2} \\
& +123 \eta_{0,1}+426 \eta_{1,0}-9 \eta_{2,-1}+87 \eta_{3,-2}-6 \eta_{4,-3} \\
& \left.\left.-213 \eta_{6,-5}+144 \eta_{8,-7}\right)\right\}+27 A_{11}\left(A_{2,2}^{S R P} / 8 A_{12}\right)^{2} \\
& \times\left\{-\left(1+C_{\varepsilon}^{2}+2 S_{\varepsilon}^{2}\right)\left(-3 \eta_{2,4}-10 \eta_{0,6}+10 \eta_{-1,7}\right.\right. \\
& \left.+12 \eta_{1,5}\right)+\left(1+C_{\varepsilon}^{2}-12 S_{\varepsilon}^{2}\right) \eta_{1,5}+15\left(1+C_{\varepsilon}^{2}\right. \\
& \left.\left.-2 S_{\varepsilon}^{2}\right)\right\} H^{2}\left(-3 \eta_{1,7}+4 \eta_{-1,9}\right)+\left[1 0 H ^ { 2 } \left(1+C_{\varepsilon}^{2}\right.\right. \\
& \left.-12 S_{\varepsilon}^{2}\right)\left(\eta_{2,6}-12 \eta_{1,7}\right)+42\left(1+C_{\varepsilon}^{2}+6 S_{\varepsilon}^{2}\right) \\
& \left.\times H^{2}\left(\eta_{0,8}+8 \eta_{-1,9}\right)\right]+5\left(1+C_{\varepsilon}^{2}-2 S_{\varepsilon}^{2}\right) H^{4} \\
& \left.\times\left(-21 \eta_{2,8}-36 \eta_{0,10}+750 \eta_{-1,11}\right)\right\}
\end{aligned}
$$$$
\mathrm{D}_{3, H}^{\ominus}=3 B_{3,2} C\left(\frac{21}{2} \eta_{3,7}-\frac{25}{4} \eta_{5,5}+\frac{9}{2} \eta_{4,6}-\frac{25}{4} \eta_{6,4}\right)
$$$$
+3 \frac{A_{1,2}}{\mu^{2}} B_{2} C\left(-90 \eta_{6,4}-36 \eta_{5,5}+\frac{297}{2} \eta_{4,6}+\frac{315}{2} \eta_{3,7}\right)
$$$$
+\frac{3 A_{11} A_{2,2}^{S R P}}{8 \mu^{2}}\left(\eta_{-2,0}-\eta_{0,-2}\right)^{-2}\left[10 \eta_{-4,6}+12 \eta_{-3,5}\right.
$$$$
+51 \eta_{-2,4}+228 \eta_{-1,3}-123 \eta_{0,2}-684 \eta_{1,1}
$$$$
+723 \eta_{2,0}+348 \eta_{3,-1}-705 \eta_{4,-2}+6 \eta_{6,-4}
$$$$
\left.+144 \eta_{8,-6}\right]+\frac{81 A_{11} A_{24}^{2}}{16 A_{12}^{2}}\left\{\left(1+C_{\varepsilon}^{2}+6 S_{\varepsilon}^{2}\right) \eta_{-1,7}\right.
$$$$
\mathrm{D}_{2, L L}^{\theta}=\frac{3}{64} \frac{A_{1,2}^{2}}{\mu^{2}}\left[\eta_{6,7}\left(180-3240 C^{2}+9300 C^{4}\right)\right.
$$$$
+\eta_{7,6}\left(1230-6660 C^{2}+5580 C^{4}\right)+\eta_{8,5}(1050
$$$$
\left.-3780 C^{2}+1050 C^{4}\right)+\eta_{5,8}\left(-420+1080 C^{2}\right.
$$$$
\left.\left.+4620 C^{4}\right)\right]+\frac{3}{128} A_{2,4}\left[\eta _ { 6 , 7 } \left(-900+9000 C^{2}\right.\right.
$$$$
\left.-10500 C^{4}\right)+\eta_{8,5}\left(1890-18900 C^{2}\right.
$$$$
\left.+22050 C^{4}\right) \eta_{5,8}+\left(-1260-16200 C^{2}-2300 C^{4}\right)
$$$$
\left.+\eta_{7,6}\left(1350-18900 C^{2}+28350 C^{4}\right)\right]
$$$$
+3 B_{2}\left(60 \eta_{6,1}-75 \eta_{7,0}+12 \eta_{5,2}\right)
$$$$
\mathrm{D}_{3, L L}^{\theta}=B_{3,2}\left\{\left(\frac{525}{4} \eta_{8,3}+\frac{225}{4} \eta_{7,4}-45 \eta_{6,5}-45 \eta_{5,6}\right)\right.
$$ 


$$
\begin{aligned}
& \left.+3 C^{2}\left(63 \eta_{5,6}-\frac{375}{4} \eta_{7,4}+45 \eta_{6,5}-\frac{525}{4} \eta_{8,3}\right)\right\} \\
& +\frac{A_{1,2}}{\mu^{2}} B_{2}\left\{\left(2160 \eta_{8,3}-1215 \eta_{6,5}-675 \eta_{5,6}\right)\right. \\
& \left.+3 C^{2}\left(-18900 \eta_{8,3}-540 \eta_{7,4}+1485 \eta_{6,5}+945 \eta_{5,6}\right)\right\} \\
& +\frac{A_{11} A_{2,2}^{S R P}}{4 \mu^{2}}\left\{24 \eta_{-1,0}\left(\eta_{0,-2}-3 H^{2}\right)\left(\eta_{-2,0}-\eta_{0,-2}\right)^{-4}\right. \\
& \times\left[723+5 \eta_{-6,6}-3 \eta_{-5,5}+10 \eta_{-4,4}+66 \eta_{-3,3}\right. \\
& \mathrm{D}_{3, G G}^{\theta}=B_{3,2}\left\{\frac{225}{4} \eta_{7,4}+\frac{75}{2} \eta_{6,5}-45 \eta_{5,6}-\frac{135}{2} \eta_{4,7}\right. \\
& -36 \eta_{-2,2}-456 \eta_{-1,1}+606 \eta_{1,-1}-1419 \eta_{2,-2} \\
& \left.-326 \eta_{3,-3}+717 \eta_{5,-5}+35 \eta_{6,-6}-288 \eta_{8,-8}\right] \\
& +4\left(\eta_{0,-2}-3 H^{2}\right)\left(\eta_{-2,0}-\eta_{0,-2}\right)^{-3}\left[30 \eta_{-5,6}\right. \\
& +15 \eta_{-4,5}-40 \eta_{-3,4}-528 \eta_{-2,3}+72 \eta_{-1,2}+456 \eta_{0,-1} \\
& +606 \eta_{2,-1}-2838 \eta_{3,-2}+9783 \eta_{4,-3}+3585 \eta_{6,-5} \\
& \left.-1890 \eta_{7,-6}+2304 \eta_{9,-8}\right]+\left\{4 \eta_{-1,0}\left(\eta_{0,-2}-3 H^{2}\right)\right. \\
& \times\left(\eta_{-2,0}-\eta_{0,-2}\right)^{-2}\left[723 \eta_{-2,0}+25 \eta_{-4,6}+12 \eta_{-3,5}\right. \\
& -30 \eta_{-2,4}-132 \eta_{-1,3}+36 \eta_{h_{2,2}}+1212 \eta_{3,1}-4257 \eta_{4,2} \\
& -13044 \eta_{5,-3}+4302 \eta_{7,-5}+2457 \eta_{8,-6} \\
& \left.\left.-2592 \eta_{10,-8}\right]\right\}-\left(\eta_{0,-2}-3 H^{2}\right)\left(\eta_{-2,0}-\eta_{0,-2}\right)^{-2} \\
& \times\left[-1446 \eta_{3,0}+100 \eta_{-3,6}+36 \eta_{-2,5}-60 \eta_{-1,4}\right. \\
& -132 \eta_{0,3}-3636 \eta_{4,-1}+17028 \eta_{5,-2}-65220 \eta_{6,-3} \\
& \left.\left.-30114 \eta_{8,-5}-19656 \eta_{9,-6}+25920 \eta_{11,-8}\right]\right\} \\
& +27 A_{11}\left(A_{2,2}^{S R P} / 8 A_{12}\right)^{2}\left\{-\left(1+C_{\varepsilon}^{2}+2 S_{\varepsilon}^{2}\right)\left(6 \eta_{4,3}\right.\right. \\
& \left.-60 \eta_{0,7}-6 \eta_{3,4}\right)+\left(1+C_{\varepsilon}^{2}-12 S_{\varepsilon}^{2}\right) H^{2} \\
& \left.\times\left(12 \eta_{4,5}+20 \eta_{3,6}\right)+90 H^{4}\left(1+C_{\varepsilon}^{2}-2 S_{\varepsilon}^{2}\right) \eta_{4,7}\right\} \\
& \begin{array}{r}
\left.+3 C^{2}\left(378 \eta_{3,8}-\frac{525}{4} \eta_{5,6}+\frac{252}{2} \eta_{4,7}-\frac{750}{4} \eta_{6,5}\right)\right\} \\
+\frac{A_{1,2}}{\mu^{2}} B_{2}\left\{\left(540 \eta_{6,5}-\frac{3645}{2} \eta_{4,7}-\frac{4725}{2} \eta_{3,8}\right)\right.
\end{array} \\
& \left.+3 C^{2}\left(-1350 \eta_{6,5}-756 \eta_{5,6}+4158 \eta_{4,7}+5670 \eta_{3,8}\right)\right\} \\
& -\frac{27\left(A_{2,2}^{S R P}\right)^{2}}{8 A_{12}} C_{\varepsilon}+\frac{A_{11} A_{2,2}^{S R P}}{4 \mu^{2}}\left\{2 \left(\eta_{-2,-1}+\eta_{0,-3}\right.\right. \\
& \left.-6 \eta_{0,-1} H^{2}\right)\left(\eta_{-2,0}-\eta_{0,-2}\right)^{-3}\left[30 \eta_{-5,7}+15 \eta_{-4,6}\right. \\
& -40 \eta_{-3,5}-198 \eta_{-2,4}+72 L \eta_{0,3}+456 \eta_{0,2}+606 \eta_{2,0} \\
& -2838 \eta_{3,-1}-9783 \eta_{4,-2}+215 \eta_{6,-4}+2106 \eta_{7,-5} \\
& \left.-2304 \eta_{9,-7}\right]+\left(\eta_{0,-2}-3 H^{2}\right)\left(\eta_{-2,0}-\eta_{0-2}\right)^{-2} \\
& \times\left[210 \eta_{-5,8}+90 \eta_{-4,7}-200 \eta_{-3,6}-66.12 \eta_{-2,5}\right. \\
& +216 \eta_{-1,4}+912 \eta_{0,3}+2838 \eta_{3,0} \\
& \left.+19566 \eta_{4,-1}-14340 \eta_{6,-3}-9450 \eta_{7,-4}+16128 \eta_{9,-6}\right] \\
& -2\left(\eta_{-2,0}+5 \eta_{0,-2}\right)\left(\eta_{-2,0}-3 H^{2}\right)\left(\eta_{-2,0}-\eta_{0,-2}\right)^{-4} \\
& +456 \eta_{0,1}-723 \eta_{1,0}-606 \eta_{2,-1}+1419 \eta_{3,-2} \\
& \times\left[5 \eta_{-5,6}+3 \eta_{-4,5}-10 \eta_{-3,4}-66 \eta_{-2,3}+36 \eta_{-1,2}\right. \\
& \left.+326 \eta_{4,-3}-717 \eta_{6,-5}-35 \eta_{7,-6}+288 \eta_{9,-8}\right] \\
& +2 \eta_{0,-1}\left(\eta_{-2,0}-3 H^{2}\right)\left(\eta_{-2,0}-\eta_{0-2}\right)^{-3}\left[-30 \eta_{-5,7}-15 \eta_{-4,6}\right. \\
& +40 \eta_{-3,5}+198 \eta_{-2,4}-72 \eta_{-1,3}-456 \eta_{0,2}-606 \eta_{2,0}
\end{aligned}
$$$$
\text { ISSN: 0974- } 6846
$$ 
Indian Journal of Science and Technology

$+1419.2 \eta_{z_{3}-1}+3261.3 \eta_{4,-2}-717.5 \eta_{\xi_{,-4}}-351.6 \eta_{h_{,-5}}$

$\left.\left.-30114 \eta_{8,-5}-19656 \eta_{9,-6}+25920 \eta_{11,-8}\right]\right\}$

$\left.+288.8 \eta_{9,-7}\right]+\frac{8}{4} \eta_{0,-1}\left(\eta_{-2,0}-\eta_{0-2}\right)^{-4}\left[-100 \eta_{-6,6}\right.$

$-18.4 \eta_{-5,5}+21.3 \eta_{-4,4}-168 \eta_{-3,3}+102 \eta_{-2,2}-600$

$-360 \eta_{1,-1}+4230 \eta_{2,-2}+696 \eta_{3,-3}-3615 \eta_{4,-4}$

$-3948 \eta_{6,-6}+3888 \eta_{8,-8}+6 H^{2}\left(105 \eta_{-6,8}\right.$

$+90 \eta_{-5,7}+155 \eta_{-4,6}+600 \eta_{-3,5}-414 \eta_{-2,4}$

$-912 \eta_{-1,3}+123 \eta_{0,2}+9 \eta_{2,0}-156 \eta_{3,-1}+18 \eta_{4,-2}$

$\left.\left.+1065 \eta_{6,-4}-1008 \eta_{8,-6}\right)\right]-600 \eta_{0,-1}-180 \eta_{1,-2}$

$+1410 \eta_{2,-3}+174 \eta_{3,-4}-723 \eta_{4,-5}-564 \eta_{6,-7}$

$+432 \eta_{8,-9}-6 H^{2}\left(15 \eta_{-6,7}+15 \eta_{-5,6}+3 \eta_{-4,5}\right.$

$+150 \eta_{-3,4}-138 \eta_{-2,3}-456 \eta_{-1,2}+123 \eta_{0,1}$

$+426 \eta_{1,0}-9 \eta_{2,-1}+87 \eta_{3,-2}-6 \eta_{4,-3}-213 \eta_{6,-5}$

$\left.\left.+144 \eta_{8,-7}\right)\right]+\frac{1}{2}\left(\eta_{-2,0}-\eta_{0-2}\right)^{-2}\left[600 \eta_{-6,7}\right.$

$+360 \eta_{-5,5}-252 \eta_{-4,5}+504 \eta_{-3,4}-204 \eta_{-2,3}$

$-360 \eta_{1,0}+8460 \eta_{2,-1}+2088 \eta_{3,-2}-14460 \eta_{4,-3}$

$-23688 \eta_{6,-5}+30456 \eta_{8,-7}-6 H^{2}\left(840 \eta_{-6,9}\right.$

$-630 \eta_{-5,8}-930 \eta_{-4,7}-3000 \eta_{-3,6}+1656 \eta_{-2,5}$

$+2736 \eta_{-1,4}+246 \eta_{0,3}+174 \eta_{3,0}-36 \eta_{4,-1}$

$\left.\left.-4260 \eta_{6,-3}+6048 \eta_{8,-5}\right)\right]-600-360 \eta_{1,-1}$

$+4230 \eta_{2,-2}+696 \eta_{3,-3}-3615 \eta_{4,-4}-3948 \eta_{6,-6}$

$+380 \eta_{8,-8}-6 H^{2}\left(-105 \eta_{-6,8}-90 \eta_{-5,7}-155 \eta_{-4,6}\right.$

$-600 \eta_{-3,5}+414 \eta_{-2,4}+912 \eta_{-1,3}-123 \eta_{0,2}-9 \eta_{2,0}$

$\left.\left.\left.+174 \eta_{3,-1}-18 \eta_{4,-2}-1065 \eta_{6,-4}+1008 \eta_{8,-6}\right)\right]\right\}$

$+27 A_{11}\left(A_{2,2}^{S R P} / 8 A_{12}\right)^{2}\left\{\left(1+C_{\varepsilon}^{2}+2 S_{\varepsilon}^{2}\right)\right.$

$\times\left(60 \eta_{-1,8}+60 \eta_{1,6}-12 \eta_{2,5}-60 \eta_{0,7}\right)$

$+\left[2\left(1+C_{\varepsilon}^{2}-12 S_{\varepsilon}^{2}\right) H^{2}\left(420 \eta_{1,8}-30 \eta_{2,7}\right)\right.$

$\left.-\left(1+C_{\varepsilon}^{2}+6 S_{\varepsilon}^{2}\right) H^{2}\left(328 \eta_{0,9}+3024 \eta_{-1,10}\right)\right]$
Vol. 5 No. 9 (Sep. 2012)

ISSN: 0974- 6846

$\left.+5\left(1+C_{\varepsilon}^{2}-2 S_{\varepsilon}^{2}\right) H^{4}\left(168 \eta_{2,9}+360 \eta_{0,11}+8250 \eta_{-1,12}\right)\right\}$ $\mathrm{D}_{3, H H}^{\ominus}=3 B_{3,2}\left(\frac{21}{2} \eta_{3,8}-\frac{25}{4} \eta_{5,6}+\frac{9}{2} \eta_{4,7}-\frac{25}{4} \eta_{6,5}\right)$

$+3 \frac{A_{1,2}}{\mu^{2}} B_{2}\left(-90 \eta_{6,3}-36 \eta_{5,4}+\frac{297}{2} \eta_{4,5}+\frac{315}{2} \eta_{3,6}\right)$

$\left.+30 H\left(1+C_{\varepsilon}^{2}-2 S_{\varepsilon}^{2}\right)\left(-3 \eta_{1,7}+4 \eta_{-1,9}\right)\right\}$

$\mathrm{D}_{2, L G}^{\theta}=\frac{3}{64} \frac{A_{1,2}^{2}}{\mu^{2}}\left[\eta_{5,8}\left(252-5832 C^{2}+20460 C^{4}\right)\right.$

$+\eta_{6,7}\left(1230-8880 C^{2}+9300 C^{4}\right)$

$+\eta_{7,6}\left(150-3780 C^{2}+1350 C^{4}\right)$

$\left.+\eta_{4,9}\left(-84+2700 C^{2}+13860 C^{4}\right)\right]$

$+\frac{3 A_{2,4}}{128}\left[\eta_{5,8}\left(-1260+16200 C^{2}-23100 C^{4}\right)\right.$

$+\eta_{7,6}\left(1350-6750 C^{2}+28350 C^{4}\right)$

$-\eta_{4,9}\left(2520-40500 C^{2}+69300 C^{4}\right)$

$\left.+\eta_{6,7}\left(1350-25200 C^{2}+47250 C^{4}\right)\right]$

$+3 B_{2}\left(12 \eta_{5,2}+6 \eta_{4,3}\right)$

$\mathrm{D}_{3, L G}^{\theta}=B_{3,2}\left\{\left(\frac{225}{4} \eta_{7,4}+\frac{75}{2} \eta_{6,5}-45 \eta_{5,6}-\frac{135}{2} \eta_{4,7}\right)\right.$

$\left.+3 C^{2}\left(\frac{189}{2} \eta_{4,7}-\frac{375}{4} \eta_{6,5}+63 \eta_{5,6}-\frac{375}{4} \eta_{7,4}\right)\right\}$

$+\frac{A_{1,2}}{\mu^{2}} B_{2}\left\{\left(810 \eta_{7,4}-1215 \eta_{5,6}+\frac{2025}{2} \eta_{4,7}\right)\right.$

$\left.+3 C^{2}\left(-1350 \eta_{7,4}-540 \eta_{6,5}+2079 \eta_{5,6}+1890 \eta_{4,7}\right)\right\}$

$-\frac{27\left(A_{2,2}^{S R P}\right)^{2}}{8 A_{12}} C_{\varepsilon}+\frac{A_{11} A_{2,2}^{S R P}}{4 \mu^{2}}\left\{8 G\left(L^{2}+2 G^{2}\right.\right.$

$\left.-9 H^{2}\right)\left(\eta_{-2,0}-\eta_{0-2}\right)^{-4}\left[-723+5 \eta_{-6,6}+3 \eta_{-5,5}\right.$

$-10 \eta_{-4,4}-66 \eta_{-3,3}+36 \eta_{-2,2}+456 \eta_{-1,1}-606 \eta_{1,-1}$

$+1419 \eta_{2,-2}+326 \eta_{3,-3}-717 \eta_{5,-5}-35 \eta_{6,-6}$

$\left.+288 \eta_{8,-8}\right]+4\left(\eta_{0,-2}-3 H^{2}\right)\left(\eta_{-2,0}-\eta_{0-2}\right)^{-3}$

$\times\left[-30 \eta_{-6,7}-15 \eta_{-5,6}+40 \eta_{-4,5}+66.3 \eta_{-3,4}\right.$

$-72 \eta_{-2,3}+12 \eta_{-3,5}-30 \eta_{-2,4}-132 \eta_{-1,3}$

$+36 \eta_{0,2}+1212 \eta_{3,-1}-4257 \eta_{4,-2}-1304 \eta_{5,-3}$ 
Indian Journal of Science and Technology

$$
\begin{aligned}
& +120 \eta_{-2,5}+396 \eta_{-1,4}-72 \eta_{0,3}+1212 \eta_{3,0} \\
& -8514 \eta_{4,-1}-39123 \eta_{5,-2}+21510 \eta_{7,-4} \\
& \left.\left.+14742 \eta_{8,-5}-20736 \eta_{10,-7}\right]\right\} \\
& +\frac{27 A_{11}\left(A_{2,2}^{S R P}\right)^{2}}{64 A_{12}^{2}}\left\{-\left(1+C_{\varepsilon}^{2}+2 S_{\varepsilon}^{2}\right)\left(6 \eta_{3,4}\right.\right. \\
& \left.-60 \eta_{0,7}\right)\left(-12 \eta_{2,5}\right)+\left(1+C_{\varepsilon}^{2}-12 S_{\varepsilon}^{2}\right) H^{2} \\
& \times\left(20 \eta_{3,6}+60 \eta_{2,7}\right)+336 H^{2}\left(1+C_{\varepsilon}^{2}+6 S_{\varepsilon}^{2}\right) \eta_{0,9} \\
& \left.+H^{4}\left(1+C_{\varepsilon}^{2}-2 S_{\varepsilon}^{2}\right)\left(210 \eta_{3,8}+750 \eta_{0,11}\right)\right\} \\
& \mathrm{D}_{3, G H}^{\theta}=B_{3,2}\left\{3 C\left(\frac{75}{2} \eta_{5,6}-84 \eta_{3,8}-\frac{63}{2} \eta_{4,7}+\frac{125}{2} \eta_{6,5}\right)\right\} \\
& +\frac{A_{1,2} B_{2}}{\mu^{2}}\left\{3 C\left(450 \eta_{6,5}+216 \eta_{5,6}-\frac{2079}{2} \eta_{4,7}-1260 \eta_{3,8}\right)\right\} \\
& -66 \eta_{-2,3}+36 \eta_{-1,2}+456 \eta_{0,1}-723 \eta_{1,0}-606 \eta_{2,-1} \\
& +1419 \eta_{3,-2}+326 \eta_{4,-3}-717 \eta_{6,-5}-35 \eta_{7,-6} \\
& \left.+288 \eta_{9,-8}\right]+456 \eta_{0,1}-723 \eta_{1,0}-606 \eta_{2,-1}+1419 \eta_{3,-2} \\
& \left.+326 \eta_{4,-3}-717 \eta_{6,-5}-35 \eta_{7,-6}+288 \eta_{9,-8}\right]+6 H \\
& \times\left(\eta_{-2,0}-\eta_{0-2}\right)^{-2}\left(-105 \eta_{-6,8}-90 \eta_{-5,7}-155 \eta_{-4,6}\right. \\
& -600 \eta_{-3,5}+414 \eta_{-2,4}+912 \eta_{-1,3}-123 \eta_{0,2}-9 \eta_{2,0} \\
& -138 \eta_{-2,3}-456 \eta_{-1,2}+123 \eta_{0,1}+426 \eta_{1,0} \\
& \left.\left.-9 \eta_{2,-1}+87 \eta_{3,-2}-6 \eta_{4,-3}-213 \eta_{6,-5}+144 \eta_{8,-7}\right)\right\} \\
& +\frac{27 A_{11}\left(A_{2,2}^{S R P}\right)^{2}}{64 A_{12}^{2}}\left\{H \left[4\left(1+C_{\varepsilon}^{2}-12 S_{\varepsilon}^{2}\right)\right.\right. \\
& \times\left(5 \eta_{2,6}-60 \eta_{1,7}\right)-84\left(1+C_{\varepsilon}^{2}+6 S_{\varepsilon}^{2}\right) \\
& \left.\times\left(-\eta_{0,8}-8 \eta_{-1,9}\right)\right]+20\left(1+C_{\varepsilon}^{2}-2 S_{\varepsilon}^{2}\right) \\
& \left.\times H^{3}\left(-21 \eta_{2,8}-36 \eta_{0,10}+750 \eta_{-1,11}\right)\right\} \\
& \mathrm{D}_{3, H L}^{\theta}=3 B_{3,2} C\left(-\frac{63}{2} \eta_{4,7}+\frac{125}{4} \eta_{6,5}-18 \eta_{5,6}+\frac{125}{2} \eta_{7,4}\right) \\
& +3 \frac{A_{1,2} B_{2}}{\mu^{2}} C\left(540 \eta_{7,4}+180 \eta_{6,5}-558 \eta_{5,6}-\frac{945}{2} \eta_{3,7}\right) \\
& -\frac{3 A_{11} A_{2,2}^{S R P}}{2 \mu^{2}} \eta_{0,-1}\left(\eta_{-2,0}-\eta_{0-2}\right)^{-3}\left[10 \eta_{-4,6}\right. \\
& +12 \eta_{-3,5}+5 \eta_{-2,4}+228 \eta_{-1,3}-123 \eta_{0,2}-684 \eta_{1,1} \\
& \left.+723 \eta_{2,0}+348 \eta_{3,-1}-705 \eta_{4,-2}+6 \eta_{6,-4}+144 \eta_{8,-6}\right] \\
& +\left(\eta_{-2,0}-\eta_{0-2}\right)^{-2}\left[40 \eta_{-3,6}+36 \eta_{-2,5}+102 \eta_{-1,4}+228 \eta_{0,3}\right.
\end{aligned}
$$

Vol. 5 No. 9 (Sep. 2012)

ISSN: 0974- 6846

$+684 \eta_{2,1}-1446 \eta_{3,0}+1044 \eta_{4,-1}+2820 \eta_{5,-2}$

$\left.-36 \eta_{7,-4}-1152 \eta_{9,-6}\right]+\frac{81 A_{11} A_{24}^{2}}{16 A_{12}^{2}}$

$\times\left\{\left(1+C_{\varepsilon}^{2}+6 S_{\varepsilon}^{2}\right) \eta_{0,7}-\left(1+C_{\varepsilon}^{2}-12 S_{\varepsilon}^{2}\right) \eta_{2,5}\right.$

$\left.+5\left(1+C_{\varepsilon}^{2}-2 S_{\varepsilon}^{2}\right) H^{2}\left(9 \eta_{2,7}+12 \eta_{0,9}\right)\right\}$

$\mathrm{D}_{3, L}^{h}=6 B_{3,2} C\left(\frac{9}{4} \eta_{4,6}-\frac{25}{8} \eta_{6,4}\right)+6 \frac{A_{1,2} B_{2}}{\mu^{2}} C$

$\times\left(\frac{135}{4} \eta_{4,6}+2 \eta_{5,5}-45 \eta_{6,4}\right)-\frac{54 C_{\varepsilon} \eta_{-1,0}\left(A_{2,2}^{S R P}\right)^{2}}{16 A_{12}}$

$-\frac{3 A_{11} A_{2,2}^{S R P}}{2 \mu^{2}}\left\{H\left(\eta_{-2,0}-\eta_{0-2}\right)^{-3}\left[10 \eta_{-5,6}+12 \eta_{-4,5}\right.\right.$

$+5 \eta_{-3,4}+228 \eta_{-2,3}-123 \eta_{-1,2}-684 \eta_{0,1}+723 \eta_{1,0}$

$\left.+348 \eta_{2,-1}-705 \eta_{3,-2}+6 \eta_{5,-4}+144 \eta_{7,-6}\right]$

$+H\left(\eta_{-2,0}-\eta_{0-2}\right)^{-2}\left[40 \eta_{-3,6}+36 \eta_{-2,5}\right.$

$+102 \eta_{-1,4}+228 \eta_{0,3}+684 \eta_{2,1}-1446 \eta_{3,0}$

$\left.\left.-1044 \eta_{4,-1}+2820 \eta_{5,-2}-36 \eta_{7,-4}-1152 \eta_{9,-6}\right]\right\}$

$+\frac{81 A_{11} A_{24}^{2}}{16 A_{12}^{2}}\left\{\left(1+C_{\varepsilon}^{2}+6 S_{\varepsilon}^{2}\right) H \eta_{0,7}-\left(1+C_{\varepsilon}^{2}-12 S_{\varepsilon}^{2}\right)\right.$

$\left.\times H \eta_{2,5}+5\left(1+C_{\varepsilon}^{2}-2 S_{\varepsilon}^{2}\right) H^{3}\left(3 \eta_{2,7}+4 \eta_{0,9}\right)\right\}$

$\mathrm{D}_{3, G}^{h}=6 B_{3,2} C\left(\frac{21}{4} \eta_{3,7}-\frac{25}{8} \eta_{5,5}\right)+6 \frac{A_{1,2}}{\mu^{2}} B_{2} C$

$\times\left(\frac{315}{4} \eta_{3,7}+\frac{162}{4} \eta_{4,6}-54 \eta_{5,5}\right)+\frac{3 A_{11} A_{2,2}^{S R P}}{2 \mu^{2}}$

$\times\left\{-H \eta_{0,-1}\left(\eta_{-2,0}-\eta_{0-2}\right)^{-3}\left[10 \eta_{-4,6}+12 \eta_{-3,5}\right.\right.$

$+5 \eta_{-2,4}+228 \eta_{-1,3}-123 \eta_{0,2}-684 \eta_{1,1}$

$\left.+723 \eta_{2,0}+348 \eta_{3,-1}-705 \eta_{-4,2}+6 \eta_{6,-4}+144 \eta_{8,-6}\right]$

$+H\left(\eta_{-2,0}-\eta_{0-2}\right)^{-2}\left[-60 \eta_{-4,7}-60 \eta_{-3,6}-204 \eta_{-2,5}\right.$

$-684 \eta_{-1,4}+246 \eta_{0,3}+684 \eta_{1,2}+348 \eta_{3,0}$

$\left.\left.-1410 \eta_{4,-1}+24 \eta_{6,3}+864 \eta_{8,-5}\right]\right\}+\frac{81 A_{11} A_{24}^{2}}{16 A_{12}^{2}}$

$\times\left\{-7\left(1+C_{\varepsilon}^{2}+6 S_{\varepsilon}^{2}\right) H \eta_{-1,8}-5\left(1+C_{\varepsilon}^{2}-12 S_{\varepsilon}^{2}\right)\right.$

$\left.\times H \eta_{1,6}+5\left(1+C_{\varepsilon}^{2}-2 S_{\varepsilon}^{2}\right) H^{3}\left(3 \eta_{2,7}-40 \eta_{-1,10}\right)\right\}$ 


$$
\begin{aligned}
& \mathrm{D}_{3, H}^{h}=-6 B_{3,2}\left(\frac{3}{4} \eta_{3,7}-\frac{5}{8} \eta_{5,5}\right)-6 \frac{A_{1,2}}{\mu^{2}} B_{2}\left(\frac{45}{4} \eta_{3,7}\right. \\
& \left.+\frac{27}{4} \eta_{4,6}-9 \eta_{5,5}\right)+\frac{3 A_{11} A_{2,2}^{S R P}}{8 \mu^{2}}\left(\eta_{-2,0}-\eta_{0-2}\right)^{-2} \\
& \times\left[10 \eta_{-4,6}+12 \eta_{-3,5}+5 \eta_{-2,4}+228 \eta_{-1,3}-123 \eta_{0,2}\right. \\
& -684 \eta_{1,1}+723 \eta_{2,0}+348 \eta_{3,-1}-705 \eta_{4,-2}+6 \eta_{6,-4} \\
& \left.+144 \eta_{8,-6}\right]+\frac{81 A_{11} A_{24}^{2}}{16 A_{12}^{2}}\left\{\left(1+C_{\varepsilon}^{2}+6 S_{\varepsilon}^{2}\right) \eta_{-1,7}\right. \\
& +\left(1+C_{\varepsilon}^{2}-12 S_{\varepsilon}^{2}\right) \eta_{1,5}+15 H^{2}\left(1+C_{\varepsilon}^{2}-2 S_{\varepsilon}^{2}\right) \\
& \left.\times\left(-3 \eta_{1,7}+4 \eta_{-1,9}\right)\right\}+\frac{81 A_{11} A_{24}^{2}}{16 A_{12}^{2}}\left\{\left(1+C_{\varepsilon}^{2}+6 S_{\varepsilon}^{2}\right) \eta_{-1,7}\right. \\
& +\left(1+C_{\varepsilon}^{2}-12 S_{\varepsilon}^{2}\right) \eta_{1,5}+15 H^{2}\left(1+C_{\varepsilon}^{2}-2 S_{\varepsilon}^{2}\right) \\
& \left.\times\left(1+C_{\varepsilon}^{2}-2 S_{\varepsilon}^{2}\right)\left(-3 \eta_{1,7}+4 \eta_{-1,9}\right)\right\} \\
& \mathrm{D}_{3, G H}^{h}=6 B_{3,2} C\left(\frac{21}{4} \eta_{3,7}-\frac{25}{8} \eta_{5,5}\right)+6 \frac{A_{1,2} B_{2}}{\mu^{2}} C \\
& \times\left(\frac{315}{4} \eta_{3,7}+\frac{162}{4} \eta_{4,6}-54 \eta_{5,5}\right)+\frac{3 A_{11} A_{2,2}^{S R P}}{2 \mu^{2}} \\
& \times\left(\eta_{-2,-1}-\eta_{0,-3}\right)^{-3} \times\left[10 \eta_{-4,6}+12 \eta_{-3,5}+5 \eta_{-2,4}\right. \\
& +228 \eta_{-1,3}-123 \eta_{0,2}-684 \eta_{1,1}+723 \eta_{2,0} \\
& +\left(\eta_{-2,0}-\eta_{0-2}\right)^{-2}\left[-60 \eta_{-4,7}-60 \eta_{-3,6}-204 \eta_{-2,5}\right. \\
& -684 \eta_{-1,4}+246 \eta_{0,3}+684 \eta_{1,2}+348 \eta_{3,0} \\
& \left.-1410 \eta_{4,-1}+24 \eta_{6,-3}+864 \eta_{8,-5}\right]+\frac{81 A_{11} A_{24}^{2}}{16 A_{12}^{2}} \\
& \times\left\{-7\left(1+C_{\varepsilon}^{2}+6 S_{\varepsilon}^{2}\right) \eta_{-1,8}-5\left(1+C_{\varepsilon}^{2}-12 S_{\varepsilon}^{2}\right) \eta_{1,6}\right. \\
& \left.+15 H^{2}\left(1+C_{\varepsilon}^{2}-2 S_{\varepsilon}^{2}\right)\left(3 \eta_{2,7}-40 \eta_{-1,10}\right)\right\} \\
& \mathrm{D}_{3, L L}^{h}=-6 B_{3,2} C\left(9 \eta_{5,6}-\frac{75}{4} \eta_{7,4}\right)-6 \frac{A_{1,2}}{\mu^{2}} B_{2} C \\
& \times\left(135 \eta_{5,6}+135 \eta_{6,5}-270 \eta_{7,4}\right)-\frac{27 C_{\varepsilon}\left(A_{2,2}^{S R P}\right)^{2}}{8 A_{12}} \\
& +\frac{3 A_{11} A_{2,2}^{S R P}}{8 \mu^{2}}\left\{-4 H\left(\eta_{-2,0}-\eta_{0-2}-6 L^{3}\right)\right. \\
& \times\left(\eta_{-2,0}-\eta_{0-2}\right)^{-4}\left[10 \eta_{-4,6}+12 \eta_{-3,5}+5 \eta_{-2,4}\right. \\
& +228 \eta_{-1,3}-123 \eta_{0,2}-684 \eta_{1,1}+723 \eta_{2,0}+348 \eta_{3,-1} \\
& \left.-705 \eta_{4,-2}+6 \eta_{6,-4}+144 \eta_{8,-6}\right]-8 H\left(\eta_{-2,0}-\eta_{0,-2}\right)^{-3} \\
& \times\left[40 \eta_{-4,6}+36 \eta_{-3,5}+102 \eta_{-2,4}+228 \eta_{-1,3}\right. \\
& +684 \eta_{1,1} 1446 \eta_{2,0}-1044 \eta_{3,-1}-2820 \eta_{4,-2} \\
& \left.-36 \eta_{6,-4}-1152 \eta_{8,-6}\right]+H\left(\eta_{-2,0}-\eta_{0-2}\right)^{-2} \\
& {\left[120 \eta_{-2,6}+72 \eta_{-1,5}-762 \eta_{0,4}-1368 \eta_{3,1}\right.} \\
& +4338 \eta_{4,0}+4137 \eta_{5,-1}+14100 \eta_{6,-2}+252 \eta_{8,-4} \\
& \left.\left.+10368 \eta_{10,-6}\right]\right\}+\frac{81 A_{11} A_{24}^{2}}{8 A_{12}^{2}}\left\{\left(1+C_{\varepsilon}^{2}-12 S_{\varepsilon}^{2}\right)\right. \\
& \left.\varlimsup_{3,5} H-30\left(1+C_{\varepsilon}^{2}-2 S_{\varepsilon}^{2}\right) H \eta_{3,7}^{3}\right\}+\frac{81 A_{11} A_{24}^{2}}{8 A_{12}^{2}} \\
& \times\left\{\left(1+C_{\varepsilon}^{2}-12 S_{\varepsilon}^{2}\right) \eta_{3,5} H-30\left(1+C_{\varepsilon}^{2}-2 S_{\varepsilon}^{2}\right) H^{3} \eta_{3,7}\right\} \\
& \mathrm{D}_{3, G G}^{h}=-6 B_{3,2} C\left(\frac{21}{4} \eta_{3,8}-\frac{25}{8} \eta_{5,6}\right)-6 \frac{A_{1,2}}{\mu^{2}} B_{2} C \\
& \times\left(\frac{315}{4} \eta_{3,8}+\frac{162}{4} \eta_{4,7}-54 \eta_{5,6}\right)+\frac{3 A_{11} A_{2,2}^{S R P}}{8 \mu^{2}} \\
& \times\left\{-4 H\left(\eta_{-2,0}-7 \eta_{0-2}\right)\left(\eta_{-2,0}-\eta_{0-2}\right)^{-4}\left[10 \eta_{-4,6}\right.\right. \\
& +12 \eta_{-3,5}+5 \eta_{-2,4}+228 \eta_{-1,3}-123 \eta_{0,2}-684 \eta_{1,1} \\
& \left.+723 \eta_{2,0}+348 \eta_{3,-1}-705 \eta_{4,-2}+6 \eta_{6,-4}+144 \eta_{8,-6}\right] \\
& -4 H\left[\left(\eta_{-2,0}-\eta_{0-2}\right)^{-3}-\left(\eta_{-2,0}-\eta_{0-2}\right)^{-2}\right] \\
& \times\left[-60 \eta_{-4,6}-60 \eta_{-3,5}-204 \eta_{-2,4}-684 \eta_{-1,3}\right. \\
& +246 \eta_{0,2}+684 \eta_{1,1}+348 \eta_{3,-1}-1410 \eta_{4,-2} \\
& \left.+24 \eta_{6,-4}+864 \eta_{8,-6}\right]+H\left(\eta_{-2,0}-\eta_{0-2}\right)^{-2} \\
& {\left[240 \eta_{-4,8}+360 \eta_{-3,7}+1020 \eta_{-2,6}+3456 \eta_{-1,5}\right.} \\
& +H\left(\eta_{-2,0}-\eta_{0-2}\right)^{-2}\left[240 \eta_{-4,8}+360 \eta_{-3,7}\right. \\
& +1020 \eta_{-2,6}+3456 \eta_{-1,4}-738 \eta_{0,4}-1368 \eta_{1,3} \\
& \left.\left.-1410 \eta 4,0+72 \eta_{6,-2}+4320 \eta_{8,-4}\right]\right\} \\
& +\frac{567 A_{11} A_{24}^{2}}{2 A_{12}^{2}}\left\{\left(1+C_{\varepsilon}^{2}+6 S_{\varepsilon}^{2}\right) H \eta_{-1,9}\right. \\
& +30\left(1+C_{\varepsilon}^{2}-12 S_{\varepsilon}^{2}\right) H \eta_{1,7}-5\left(1+C_{\varepsilon}^{2}\right. \\
& \left.\left.-2 S_{\varepsilon}^{2}\right)\left(168 \eta_{1,9}+360 \eta_{-1,11}\right) H^{3}\right\}
\end{aligned}
$$




$$
\begin{aligned}
& \mathrm{D}_{3, L G}^{h}=-6 B_{3,2 C}\left(\frac{63}{4} \eta_{4,7}-\frac{225}{8} \eta_{6,5}\right)-6 \frac{A_{1,2} B_{2}}{\mu^{2}} C \\
& \times\left(\frac{945}{4} \eta_{4,7}+162 \eta_{5,6}-225 \eta_{6,5}\right)+\frac{3 A_{11} A_{2,2}^{S R P}}{8 \mu^{2}} \\
& \times\left\{-24 H\left(\eta_{-2,0}-\eta_{0,-2}\right)^{-4}\left[10 \eta_{-5,5}+12 \eta_{-4,4}\right.\right. \\
& +51 \eta_{-3,3}+228 \eta_{-2,2}-123 \eta_{-1,1}-684+723 \eta_{1,-1} \\
& \left.+348 \eta_{2,-2}-705 \eta_{3,-3}+6 \eta_{5,-5}+144 \eta_{7,-7}\right]-4 H \\
& \left(\eta_{-2,0}-\eta_{0,-2}\right)^{3}\left[-60 \eta_{-5,7}-60 \eta_{-4,6}-204 \eta_{-3,5}\right. \\
& -684 \eta_{-2,4}+246 \eta_{-1,3}+684 \eta_{, 2}-2454 \eta_{3,-1} \\
& \left.+24 \eta_{5,-3}+864 \eta_{7,-5}\right]-4 H\left(\eta_{-2,0}-\eta_{0,-2}\right)^{-3} \\
& \times\left[40 \eta_{-3,5}+36 \eta_{-2,4}+102 \eta_{-1,3}+228 \eta_{0,2}+684 \eta_{2,0}\right. \\
& -1446 \eta_{3,-1}-1044 \eta_{4,-2}-2820 \eta_{5,-3}-36 \eta_{7,-5} \\
& \left.-1152 \eta_{9,-7}\right]+H\left(\eta_{-2,0}-\eta_{0,-2}\right)^{2}\left[-240 \eta_{-3,7}-180 \eta_{-2,6}\right. \\
& -408 \eta_{-1,5}-864 \eta_{0,4}-684 \eta_{2,2}-1044 \eta_{4,0} \\
& \left.\left.-5640 \eta_{5,-1}-144 \eta_{7,-3}-6912 \eta_{9,-5}\right]\right\} \\
& -\frac{567 A_{11} A_{24}^{2}}{16 A_{12}^{2}}\left\{\left(1+C_{\varepsilon}^{2}+6 S_{\varepsilon}^{2}\right) H \eta_{0,8}\right. \\
& +5\left(1+C_{\varepsilon}^{2}-12 S_{\varepsilon}^{2}\right) H \eta_{2,6}-5\left(1+C_{\varepsilon}^{2}-2 S_{\varepsilon}^{2}\right) \\
& \left.\times H^{3}\left(21 \eta_{2,8}+36 \eta_{0,10}\right)\right\} \\
& +15 H^{2}\left(1+C_{\varepsilon}^{2}-2 S_{\varepsilon}^{2}\right)\left(3 \eta_{2,7}+4 \eta_{0,9}\right) \\
& \mathrm{D}_{3, H L}^{h}=6 B_{3,2}\left(\frac{9}{4} \eta_{4,7}-\frac{25}{8} \eta_{6,5}\right)+6 \frac{A_{1,2}}{\mu^{2}} B_{2} \\
& \times\left(\frac{135}{4} \eta_{4,7}+27 \eta_{5,6}-45 \eta_{6,5}\right)+\frac{3 A_{11} A_{2,2}^{S R P}}{8 \mu^{2}} \\
& \times\left\{-4\left(\eta_{-2,0}-\eta_{0-2}\right)^{-3}\left[10 \eta_{-5,6}+12 \eta_{-4,5}+5 \eta_{-3,4}\right.\right. \\
& +228 \eta_{-2,3}-123 \eta_{-1,2}-684 \eta_{0,1}+723 \eta_{1,0} \\
& \left.+348 \eta_{2,-1}-705 \eta_{3,-2}+6 \eta_{5,-4}+144 \eta_{7,-6}\right] \\
& +\left(\eta_{-2,0}-\eta_{0-2}\right)^{-2}\left[40 \eta_{-3,6}+36 \eta_{-2,5}+102 \eta_{-1,4}\right. \\
& +228 \eta_{0,3}+684 \eta_{2,1}-1446 \eta_{3,0}-1044 \eta_{4,-1} \\
& \left.-2820 \eta_{5,-2}-36 \eta_{7,-4}-1152 \eta_{9,-6}\right]+\frac{81 A_{11} A_{24}^{2}}{16 A_{12}^{2}} \\
& \times\left(1+C_{\varepsilon}^{2}+6 S_{\varepsilon}^{2}\right) \eta_{0,7}-\left(1+C_{\varepsilon}^{2}-12 S_{\varepsilon}^{2}\right) \eta_{2,5}
\end{aligned}
$$

Vol. 5 No. 9 (Sep. 2012)

ISSN: 0974- 6846 